NBER WORKING PAPER SERIES

\title{
NEEDLE SHARING AND HIV TRANSMISSION: A MODEL WITH MARKETS AND PURPOSIVE BEHAVIOR
}

\author{
Ajay Mahal \\ Brendan O'Flaherty \\ David E. Bloom \\ Working Paper 14823 \\ http://www.nber.org/papers/w14823
NATIONAL BUREAU OF ECONOMIC RESEARCH
1050 Massachusetts Avenue
Cambridge, MA 02138
March 2009

Earlier versions of this paper were presented at the annual meetings of the Econometric Society, the International AIDS Economics Network, seminars at the Columbia University Department of Economics and Mailman School of Public Health and the Harvard School of Public Health. The authors wish to thank David Canning, Dhaval Dave, John McLaren, Larry Rosenberg, Jaypee Sevilla, and numerous seminar participants for their comments. The views expressed herein are those of the author(s) and do not necessarily reflect the views of the National Bureau of Economic Research.

NBER working papers are circulated for discussion and comment purposes. They have not been peerreviewed or been subject to the review by the NBER Board of Directors that accompanies official NBER publications.

(C) 2009 by Ajay Mahal, Brendan O'Flaherty, and David E. Bloom. All rights reserved. Short sections of text, not to exceed two paragraphs, may be quoted without explicit permission provided that full credit, including $\odot$ notice, is given to the source. 
Needle Sharing and HIV Transmission: A Model with Markets and Purposive Behavior Ajay Mahal, Brendan O'Flaherty, and David E. Bloom

NBER Working Paper No. 14823

March 2009

JEL No. I18,K42

\begin{abstract}
$\underline{\text { ABSTRACT }}$
Without well designed empirical studies, mathematical models are an important way to use data on needle infection for inferences about human infection. We develop a model with explicit behavioral foundations to explore an array of policy interventions related to HIV transmission among IDU. In our model, needle exchanges affect the spread of HIV in three ways: more HIV-negative IDUs use new needles instead of old ones; needles are retired after fewer uses; and the proportion of HIV-positive IDUs among users of both old and new needles rises owing to sorting effects. The first and second effects reduce the long-run incidence of HIV, while the third effect works in the opposite direction. We compare the results of our model with those of Kaplan and O'Keefe (1993) that is the foundation of many later models of HIV transmission among IDU.
\end{abstract}

\section{Ajay Mahal}

Harvard School of Public Health

Department of Global Health and Population

665 Huntington Ave

Boston, MA 02115

amahal@hsph.harvard.edu

Brendan O'Flaherty

Columbia University

bo2@columbia.edu
David E. Bloom

Harvard School of Public Health

Department of Global Health and Population

665 Huntington Ave.

Boston, MA 02115

and NBER

dbloom@hsph.harvard.edu 


\section{Introduction}

Of the estimated 33 million people worldwide living with HIV currently (UNAIDS 2008), a substantial proportion are injecting drug users (IDUs). The relationship between injecting drug use and HIV is particularly strong in Eastern Europe and Central Asia, with IDUs and their sexual partners accounting for nearly two-thirds of all new cases reported with HIV in Ukraine and the Russian Federation in recent years (UNAIDS 2008). In China, it is estimated that almost half of all cases of HIV are accounted for by IDUs. IDUs are also heavily represented among people with HIV in areas of Myanmar, India and Vietnam (Tilson et al. 2007); and about one-fifth of all new HIV infections in Canada and the United States are attributed to injection drug use (UNAIDS 2007).

$\mathrm{HIV}$ is transmitted among IDUs primarily through the use of contaminated needles and syringes. How IDUs use needles and syringes, therefore, and how that behavior can be modified are major public health questions. Unprotected sex is also known to be an important risk factor in HIV transmission among IDUs, although we ignore this possibility in order to keep the discussion manageable (Vickerman and Watts 2003; Tilson et al. 2007). ${ }^{2}$

A voluminous empirical literature examines influences on needle use, particularly drug treatment and needle provision (needle exchange (NEP) and legalization) programs. A recent Institute of Medicine (IOM) review of the literature, however, reached the surprising conclusion that the impact of needle exchanges and legalization on HIV incidence and prevalence is not well understood (Tilson et al. 2007). Empirical assessment of needle provision on HIV transmission is difficult because NEPs are not usually undertaken as stand-alone interventions, but rather as a package of services, of which needle exchange is only one component. ${ }^{3}$ More generally, existing

\footnotetext{
${ }^{2}$ Other components of drug injecting paraphernalia such as cotton and the water used to rinse equipment could also be potential sources of HIV transmission (Turner, Miller, and Moses 1989).

${ }^{3}$ These include education programs for HIV prevention, referral to drug treatment services, etc.
} 
empirical assessments of needle provision are complicated by the presence of a range of confounding factors that could potentially also influence HIV transmission: social norms relating to drug use and especially injecting drug use, the attitude of the criminal justice system towards IDUs, and drug and needle prices. The IOM report recommends that a large-scale, multi-country randomized controlled trial be implemented as a way to assess the impacts of needle provision programs (Tilson et al. 2007). However, it is likely to be extremely difficult to follow up such a population after an intervention. As a consequence, such a trial will be enormously expensive and time-consuming to undertake even if we ignore the associated ethical dimensions.

An alternative approach to assessing the impact of needle provision programs is to use mathematical models of HIV transmission among IDUs. These provide a theoretical basis for predicting what the effect of different interventions should be, and they allow researchers to make inferences about conditions they cannot observe from data they do observe. Beginning with the seminal work of Kaplan (Kaplan 1989; Kaplan and O’Keefe 1993), a number of authors have tried to mathematically model HIV among IDUs and assess the implications for HIV incidence (and prevalence) of policy interventions (Blower et al. 1991; Murray et al. 2003; Long et al. 2006; Vickerman et al. 2006; Vickerman et al. 2007). Some authors have sought to assess the potential implications of hypothetical policy scenarios on HIV infection. Others, such as Kaplan and O'Keefe (1993) sought to assess the impact of a specific intervention - in their case, the New Haven needle exchange program - on HIV incidence among IDUs.

Mathematical models cannot, in any sense, prove that policy interventions work. But they can point to ways in which NEPs, for instance, could work, and what sort of data researchers could expect to find if they did work; and they can also indicate under which circumstances NEPs are most likely to be effective and under which circumstances they might be counter-productive. 
Moreover, findings from mathematical models can support empirical findings of correlations between policies and HIV outcomes among IDU as those reported in Hurley et al. (1997) and MacDonald et al. (2003). But mathematical models are useful only if they are built on reasonable assumptions about how people behave and are consonant with empirical findings in other related fields.

The major drawback with existing mathematical models of HIV transmission among IDU, however, is that they are essentially versions of models of malaria transmission. Malaria is spread when mosquitoes (randomly) bite infected humans and then (randomly) bite uninfected humans. These models treat needles like mosquitoes, injections like mosquito bites, and needles returned to an exchange program (as one example) like mosquitoes captured in a net. Extensions of this basic approach can be found in a variety of other work, including studies of Hepatitis-C transmission among IDUs and studies that incorporate sexual behavior as an additional factor influencing HIV transmission among IDUs (Pollack 2001; Vickerman et al. 2006).

Our paper is motivated by the observation that existing models of injecting drug use neglect crucial elements of IDU behavior that underlie HIV transmission. Consider the treatment of needles as analogous to mosquitoes in existing models. This is at odds with the fact that the number of needles in use depends on how people decide to buy, sell, and steal them, and is not determined exogenously. Similarly, needle injections are not like (random) mosquito bites. People decide when to get injections, and what needle to use; whereas, in malaria models, you cannot choose what mosquito bites you. Nor are needles returned to needle exchanges like mosquitoes captured in a net: somebody had to turn them in, and not wait another day to do so. To be sure, some of the existing work has noted this drawback. Vickerman et al. (2006, p.356), for instance, observe that “...in reality, it is unlikely that infected IDUs will be randomly distributed...” However, the approach in 
the existing literature has been to undertake sensitivity analyses allowing for a range of behavioral parameters, rather than to explicitly model such behavior which typically leads to co-dependence across some of the parameters that the existing literature takes as exogenously given.

All these elements of volition argue that economic analysis that takes people's intentions and behavior into account has an important role to play. Needles and syringes are bought, sold, and rented on the street frequently (Hopkins 1988, Leonard et al. 1999). Indeed, prices are quoted much more often in connection with injecting drug behavior than in connection with noncommercial sex, the other high-risk behavior that economists have studied (Kremer 1996, Philipson and Posner 1993). By focusing on behavior, economic analysis can clarify the considerable and sometimes contradictory evidence that exists on the effectiveness (or otherwise) of needle exchange programs (Leonard et al. 1999; Tilson et al. 2007). Surprisingly, though, economic research has tended to neglect the study of IDUs (Bloom et al. (2005) and Mahal 1995 are two exceptions; see also DeSimone 2002a for an empirical analysis).

We model IDU behavior that explicitly allows for intentional behavior and needle markets. By doing so we can analyze several different policies that have been proposed for reducing the spread of HIV among IDUs, such as needle exchange programs, legalization of needle sales, and legalization of needle possession. For each of these policies, we will be interested in its effects on the spread of HIV, and sometimes on the volume of drug use, since reducing one or both of these seems to be the objective pursued by both proponents and opponents of the policies we are discussing (Anderson 1991, Nadelmann et al. 1994). The self-perceived well-being of IDUs, the normal economic objective, does not figure prominently in these discussions, so we will make only passing mention of it. 
In our model, interventions like needle exchanges and sale legalization that make new, clean needles easier for IDUs to acquire affect the spread of HIV in three different ways. First, more HIV-negative IDUs use new needles instead of old ones. Second, needles are retired after fewer uses. Third, all else the same, the proportion of HIV-positive IDUs among users of both old and new needles is affected because of the 'sorting effect' introduced by the availability of needles as seen in earlier work by Philipson and Posner (1993) and Kremer (1996). The first and second effects reduce the long-run incidence of HIV, while the third effect can sometimes work in the opposite direction. When HIV is a serious problem, the first and second effects are likely to dominate.

We conduct simulations to explore the implications of NEPs and needle legalization on HIV infections among IDUs in our model. We also compared the findings from our model with predictions from one existing influential mathematical model described in the seminal work of Kaplan (1989) from which several later models have been derived in the literature (see Murray et al. 2003). It turns out that only the second effect—shorter needle life span-operates in the Kaplan model. Our simulations indicate that with plausible parameters the first effect is very large, relative to the third, so our model predicts that needle exchange programs usually cause larger decreases in HIV incidence than in the Kaplan model. But we find also find that under some circumstances NEPs cannot be expected to reduce human infection, and we delineate what those circumstances are. Our simulation results also suggest that conclusions produced by existing mathematical models about the impact of a needle exchange program on human infection are not robust.

The next section II develops a model that explicitly allows for endogenous IDU behavior assuming that there are no costs of carrying injecting equipment and drugs and that needle 
markets are perfectly competitive. ${ }^{4}$ The analysis suggests that legalizing sales of needle and syringes would have effects very similar to establishing an NEP. Section III extends this model to include carrying costs.

Section IV discusses the impact of NEPs on drug use. Section V presents our simulation results and Section VI is the concluding section.

\section{A Model of IDU Behavior with Needle Markets}

In this section we present a model of IDU behavior with needle markets. We focus first on the demand side and then on the supply side, including equilibrium in the market for needle rentals.

\section{The Demand for New and Used Needles}

All users of needles can distinguish an unused (“new”) needle from a used (“old”) needle, but none can distinguish among old needles of various ages. There are two rental prices: $\mathrm{p}_{\mathrm{n}}$ the rental price of new needles, and p, the rental price of old needles (we shall justify this assertion below). This two-price system has actually been observed in shooting galleries. ${ }^{5}$

Each IDU faces a choice each period between renting a new needle and renting an old needle. The cost to an IDU of using a new needle is the rental price $p_{n}$, which is the same for every user. The cost of using an old needle is the sum of three terms: rental price, bluntness, and infection risk. The rental price is for an old needle is p. The older a needle is, the blunter it becomes and, therefore, the more painful it is to use. Let $\beta(s)$ be the monetary equivalent of the pain caused by a

\footnotetext{
${ }^{4}$ Costs of carrying equipment include, for example, the penalties that an IDU may face for possession of injecting equipment, and the increase in the expected cost of carrying drugs when also carrying injecting equipment (see below). Moreover, keeping equipment at home appears to solve this problem at the cost of greater disharmony in the domestic arena (Des Jarlais and Friedman 1994) and increases the volume of drugs transacted during any one transaction.

${ }^{5}$ Communication with Denise Paone (Chemical Dependency Unit of the Beth Israel Medical Center, New York City)
} 
needle that has been used s times before $\left(\beta^{\prime}(s)>0\right)$ relative to the pain caused by a new needle $\beta(1)$, taken to be the same for each individual. We will work with steady states. In steady state the number of new needles in each period must equal the number retired in each period, so the number of needles of every active vintage must be the same. Let "t" denote the age at which needles are retired. Thus the expected bluntness of an old needle is:

$$
b(t)=\frac{1}{t-1} \int_{1}^{t} \beta(s) d s
$$

It can be checked that $b(t)$ is increasing in " $\mathrm{t}$ ". We assume that the expected bluntness cost is the same for everyone.

Let $\phi$ denote the expected subjective monetary cost of being infected with HIV, $\lambda$ the probability that an old needle is contaminated with HIV and $\tau$ the probability of HIV transmission when an HIV-negative person is using an infected needle..$^{6}$ Then $\lambda \tau \phi$ is the expected subjective infection cost from using an old needle. We will take $\tau$ as a parameter, and consider $\lambda$ to be endogenous.

A key assumption that we make is that $\phi$ is different for different IDUs, and it may even differ for the same IDU at different times. An IDU suffering from withdrawal may consider $\phi$ tiny, while the same person may, a day later, worry a great deal about HIV. We insist though that the subjective infection cost $\phi$ be linked to the actual HIV status of an individual and that, conditional

\footnotetext{
${ }^{6}$ Our analytical formulation and results are unaffected by the likelihood of re-infection (or super-infection) of HIV-positive IDUs by another strain of the virus as long as the cost of such re-infection is lower than the cost to an HIV-negative IDU of acquiring HIV. In general, the evidence on super-infection among IDUs is rather limited (Tsui et al. 2004). Moreover, even the evidence that is available on the subject suggests that prior HIV-infection provides some protection against infection from newer HIV strains (van der Kuyl and Cornelissen 2007).
} 
on HIV status, the distribution of costs from which $\phi$ is drawn is stable, even if no individual costs are stable. Specifically, we divide the population at any moment of time into two subsets - those who are HIV-positive and those who are not. This is an objective medical division, not a subjective one; we do not require that individuals accurately know their HIV-status. Let $H(\phi)$ denote the $c d f$ of $\phi$ in the HIV-negative population, and $J(\phi)$ denote the $c d f$ of $\phi$ in the HIV-positive population, with identical supports of $\left[\phi_{0}, \phi_{1}\right]$. We assume that these distributions are invariant to time and to all other parameter changes in the model. Notice, however, that for any individual IDU, the relevant distribution can change over time, if his HIV-status changes. We assume:

(S1): For all $\phi \in\left[\phi_{0}, \phi_{1}\right], J(\phi) \geq H(\phi)$

(Stochastic Dominance)

Let $h(\phi)$ and $j(\phi)$ denote the respective probability density functions corresponding to $H(\phi)$ and $J(\phi)$.

(S2): $\frac{h(\phi)}{j(\phi)}$ is a non-decreasing function of $\phi \quad$ (Monotone Likelihood Ratio Property)

Stochastic dominance is the formalization of the assumption that infection costs are generally less for HIV-positive IDU. This statement is likely to be strictly true about objective infection costs; we are assuming a weaker relation that subjective infection costs are not totally divorced from objective costs.

The Monotone Likelihood Ratio Property (MLRP) imposes further structure to the differences in the distribution of costs across HIV-positive and HIV-negative individuals and helps us to sign certain effects. The assumption means that if we observe a value of $\phi$ and are asked to 
assess whether the IDU is HIV-positive or not, the greater the value of $\phi$ the more likely it is that the IDU is HIV-negative.

A special case will sometimes be useful for illustrative purposes. We call this the "Lump Difference Case.” Suppose that some fraction L of HIV-positive IDU have been tested and are certain that they are HIV positive. They are also entirely selfish and care nothing about the safety of other IDUs. For all other HIV-positive IDUs, we assume that their distribution of infection costs is identical to that of HIV-negative IDUs (the other HIV-positive IDUs have no different information than HIV-negative IDUs). Then we have

$$
\begin{array}{ll}
J(\phi)=L+(1-L) H(\phi) & \text { All } \phi \\
j(\phi)=(1-L) h(\phi) & \phi>\phi_{0}
\end{array}
$$

Each IDU gets high every period, and seeks to minimize the cost of doing so (implicitly there is another desired good, such as food). Consider an IDU with subjective infection cost $\phi$. He will use an old needle if and only if the costs of doing so are less than the cost of using a new needle, that is

$$
\begin{aligned}
& p+b(t)+\lambda \tau \phi \leq p_{n}, \text { or } \\
& \phi \leq \frac{p_{n}-p-b(t)}{\lambda \tau} \equiv \phi^{*}
\end{aligned}
$$

Otherwise he will use a new needle. Thus the share of HIV-negative IDUs using new needles is $\left(1-H\left(\phi^{*}\right)\right)$ and the share of HIV-positive IDUs using new needles is $\left(1-J\left(\phi^{*}\right)\right)$, which is less than $\left(1-H\left(\phi^{*}\right)\right)$, by stochastic dominance.

Let $\pi \in(0,1)$ be the proportion of IDUs who are HIV-positive; we will later allow this variable to be endogenous. Then the proportion of IDUs using new needles is 
(4)

$$
q\left(\phi^{*}\right)=\pi\left(1-J\left(\phi^{*}\right)\right)+(1-\pi)\left(1-H\left(\phi^{*}\right)\right)
$$

Because $q\left(\phi^{*}\right)$ is the demand for new needles, the proportion of IDUs using old needles is

(5) $\quad 1-q\left(\phi^{*}\right)=\pi J\left(\phi^{*}\right)+(1-\pi) H\left(\phi^{*}\right)$

For future reference, we will need to consider the proportion of HIV-positive IDUs in each class of needle used, old, or new. Let $\alpha$ denote the proportion of users of new needles who are HIVpositive, and $\omega$ the proportion of HIV-positive IDUs among users of old needles. Then note that

(6) $\quad \alpha=\frac{\pi\left(1-J\left(\phi^{*}\right)\right)}{q\left(\phi^{*}\right)}$

(7) $\quad \omega=\frac{\pi J\left(\phi^{*}\right)}{1-q\left(\phi^{*}\right)}$

We have:

$\underline{\text { Result 1: Given Stochastic Dominance (S1), } \alpha \leq \omega}$

Proof: See Technical Appendix A.

Result 2: Given Stochastic Dominance (S1) and Monotone Likelihood Ratio Property (MLRP) (S2), $\alpha$ and $\omega$ are non-increasing in $\phi^{*}$.

Proof: See Technical Appendix A.

The monotone likelihood ratio property (MLRP) was crucial to the second result, which shows that a decline in $\phi^{*}$ raises the proportion of HIV-positive individuals among users of new 
needles. Intuitively, MLRP assures that people on the margin between using old and new needles are less likely to be HIV positive than infra-marginal old needle users but more likely than inframarginal users of new needles. If $\phi^{*}$ falls and moves some marginal IDU from old needle use to new needle use, both $\omega$ and $\alpha$ will rise: $\omega$ rises because the IDU who stop using old needles were the least likely to be HIV-positive in the old needle using group, and $\alpha$ rises because the added users of new needles are more likely to be HIV-positive than the original users of new needles.

Subsequently, we will show that if an NEP reduces HIV infection, it does so by reducing $\phi^{*}$. This requires an analysis of needle markets. Before proceeding to that, however, we demonstrate the circumstances under which a decline in $\phi^{*}$ will reduce HIV infection of needles and of humans. In particular, we will focus on how changes in $\phi^{*}$ influence the length of time a needle is used, the proportion of old needles infected, the rate of new human infections, and steadystate HIV prevalence.

\section{Length of Needle Use Time}

As noted above, in the steady state, the number of needles of each vintage must be the same. We also know that the number of new needles each period in a steady state is $q\left(\phi^{*}\right)$. Thus the number of needles of each vintage each period is also $q\left(\phi^{*}\right)$. Let " $\mathrm{t}$ " denote the number of times a needle is used as an old needle before it is retired (or discarded). Thus the number of IDU using old needles every period is $t q\left(\phi^{*}\right)$. Since the number of IDU using new needles is $q\left(\phi^{*}\right)$ and the total number of IDU is (normalized to) one, we have

$$
q\left(\phi^{*}\right)+t q\left(\phi^{*}\right)=1
$$




$$
t=\frac{1-q\left(\phi^{*}\right)}{q\left(\phi^{*}\right)}
$$

If an NEP reduces $\phi^{*}$, it must increase the users of new needles, namely $q\left(\phi^{*}\right)$, and hence result in a decrease in $t$. Indeed, any action that reduces $\phi^{*}$ also causes needles to be retired earlier.

From expression (8) we can also say something about the expected number of times a needle is used by an HIV-positive IDU in steady state, before it is retired. First, consider old needles. The expected number of times an old needle is used by HIV-positive IDUs ist $\omega$. Since reducing $\phi^{*}$ increases $\omega$ and decreasest, it is not immediately obvious how $\phi^{*}$ affects $t \omega$. However,

$$
t \omega=\frac{1-q\left(\phi^{*}\right)}{q\left(\phi^{*}\right)} \frac{\pi J\left(\phi^{*}\right)}{1-q\left(\phi^{*}\right)}=\frac{\pi J\left(\phi^{*}\right)}{q\left(\phi^{*}\right)}
$$

Decreasing $\phi^{*}$ reduces $J\left(\phi^{*}\right)$ and increases $q\left(\phi^{*}\right)$, and so reduces $t \omega$.

The expected number of times during its entire life that a needle is used by an HIV-positive IDU is $(\alpha+t \omega)$. Since reducing $\phi^{*}$ raises $\alpha$, it is again not immediately obvious how $\phi^{*}$ affects a needle’s total expected usage by HIV-positive IDUs. Note, however, that

$$
\alpha+t \omega=\frac{\pi\left(1-J\left(\phi^{*}\right)\right)}{q\left(\phi^{*}\right)}+\frac{\pi J\left(\phi^{*}\right)}{q\left(\phi^{*}\right)}=\frac{\pi}{q\left(\phi^{*}\right)}
$$

So, reducing $\phi^{*}$ reduces $(\alpha+t \omega)$. Thus, a successful NEP increases the expected number of times an HIV-positive IDU uses a needle when new, but decreases the expected number of times this occurs when the needle is old, and the latter effect is larger than the former. Collecting these results, we have 
Result 3: Under (S1) and (S2), in steady state, a decrease in $\phi^{*}$

(3a) causes needles to be retired earlier;

(3b) lowers the expected number of times a needle is used by an HIV-positive IDU.

\section{Needle Infection Rates}

The probability that a randomly chosen needle is infected with HIV involves a more complicated function than the expected number of times a needle will eventually be used by an HIV-positive IDU. Let $I(t)$ denote the proportion of age $t$ needles that are contaminated with HIV. Assume that an uncontaminated needle becomes infected with probability 1, if used by an HIVpositive IDU. ${ }^{7}$ Because old needles become infected with hazard rate $\omega$ once bleaching/virus attrition has occurred at the rate $\theta, I(t)$ must satisfy the differential equation

$$
\frac{\mathrm{d}}{\mathrm{dt}} I(t)=\omega[1-I(t)(1-\theta)]-\theta I(t) \text { subject to the initial condition } I(0)=\alpha
$$

Here $\alpha$ is the average HIV-prevalence rate among new needle users. This implies

$$
I(t)=\left(\frac{\omega}{\theta+\omega-\theta \omega}\right)+\left(\frac{\theta \alpha+\alpha \omega-\omega-\theta \alpha \omega}{\theta+\omega-\theta \omega}\right) e^{-(\theta+\omega-\theta \omega) t}
$$

It is easy to prove that this is always an increasing function of $t$.

\footnotetext{
${ }^{7}$ It is possible that some strains of HIV (especially those other than strain B of HIV-1) may not be as easily transmitted to the needle. However, we are not aware of reliable evidence on this point.
} 
Note that the probability $\lambda(t)$ that a randomly chosen old needle is infected is the average value of $I(t)$. The steady-state assumption implies all ages are equally represented. It follows that

$$
\lambda(t)=\frac{1}{t} \int_{0}^{t} I(s) d s=\lambda(t)=\left(\frac{\omega}{\theta+\omega-\theta \omega}\right)+\left(\frac{\theta \alpha+\alpha \omega-\omega-\theta \alpha \omega}{t(\theta+\omega-\theta \omega)^{2}}\right)\left(1-e^{-(\theta+\omega-\theta \omega) t}\right)
$$

For the moment, we will assume that $\theta=0$. This allows us to simplify the average and terminal needle infection rates to

$$
\begin{aligned}
& I(t)=1-(1-\alpha) e^{-t \omega} \\
& \lambda(t)=1-(1-\alpha) \frac{1-e^{-t \omega}}{t \omega}=1-(1-\alpha) g(t \omega)
\end{aligned}
$$

Here $g(t \omega)=\frac{1-e^{-t \omega}}{t \omega}$. The properties of the function $g($.$) are further explored in Technical$ Appendix A. The terminal infection rate in (14) is a simpler expression even though it has less welfare relevance, so we consider it first.

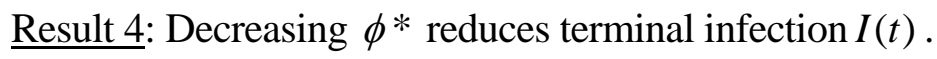

\section{Proof: See Technical Appendix A.}

We cannot make such a strong statement about the average infection rate. Under some circumstances, reducing $\phi^{*}$ increases the average infection rate $\lambda(t)$. However, we can cite three interesting cases (Results 5-7) where reducing $\phi^{*}$ reduces average infection. Detailed discussion of 
the conditions under which these relationships hold can be found in technical appendix A, along with proofs of the following results.

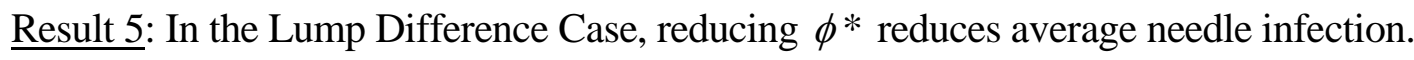

Result 6: If $t \omega>x^{*}$ then reducing $\phi^{*}$ always reduces average needle infection rates. Here $x^{*} \cong 1.79$ is the solution to the equation $e^{-x}\left(1+x+x^{2}\right)=1$.

It can be readily checked that for $x \in\left(0, x^{*}\right), e^{-x}\left(1+x+x^{2}\right)>1$ with the opposite holding true for $x>x^{*}$.

Is $t \omega>1.79$ possible? Such values seem plausible in American cities. Even if $\omega$ is very close to the overall HIV prevalence rate $\pi$, it may often be in the $30-50 \%$ range. Ethnographic studies report 5-8 uses per needle, so $t$ would be 4-7. This gives a range of 1.2-4.0 for t $\omega$.

A sufficient condition for Result 6 is that $\frac{\pi J}{q}>x^{*}=1.79$. As is clear in the appendix proof of Result 6, for smaller $\frac{\pi J}{q}$, this condition will hold when $\frac{h}{j}$ is sufficiently large: that is when the number of HIV-negative individuals who switch from old needles to new is large, relative to the number of HIV-positive who do so. Finally, we also have

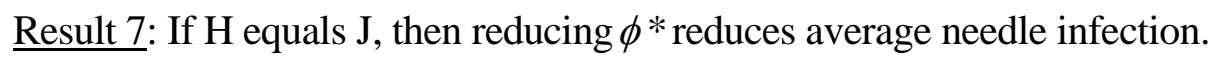

For all three cases discussed above, the desired inequality is strict. Thus the results also hold for some close enough approximations to the cases discussed, as well. When $\frac{\partial \lambda}{\partial \phi^{*}}>0$ at some particular set of parameters, we say that the average needle infection response is regular. That is, 
average needle infection response is regular in the lump-difference case, whenever $\frac{\pi J}{q}>x^{*}=1.79$ and also when $\mathrm{H}$ and $\mathrm{J}$ are identical.

\section{New Human Infections}

Human infections occur when an HIV-negative IDU uses an infected needle. Consider the variable $\mathrm{Z}$ defined as

$$
Z\left(\phi^{*}, \pi\right)=H\left(\phi^{*}\right) \lambda
$$

The term $(1-\pi) \tau Z=(1-\pi) H\left(\phi^{*}\right) \lambda \tau$ indicates the (instantaneous) incidence of HIV in a population normalized to size 1. Only HIV-negative IDUs using old needles are at risk of being infected and their number is given by $H\left(\phi^{*}\right)(1-\pi)$. Here $\tau$ is the probability of HIV infection when using an infected needle. The likelihood that an old needle is infected is $\lambda$.

A reduction in $\phi^{*}$ affects the rate of new human infections in two ways: it reduces $H\left(\phi^{*}\right)$, the number of people exposed to HIV; and it changes the average needle infection rate $\lambda$. If the average needle infection response is regular, both of these effects work in the same direction: reducing $\phi^{*}$ reduces the rate at which new humans are infected with HIV.

We cannot prove, however, that effective NEPs always reduce the rate of new human infection (see Technical appendix B for a counter example). When reductions in $\phi^{*}$ reduce Z we say that the case is normal. Obviously, regularity implies normality, but not the converse. In general, normality obtains whenever

$$
h\left\{\lambda+\frac{H}{1-H}(1-J)-H \frac{1-\pi}{q} J \frac{g^{\prime}}{g}\right\}>\frac{j H}{g}\left\{g+g^{\prime}\left(1+\frac{\pi J}{q}\right)\right\}
$$


The inequality in (17) follows from differentiating $\mathrm{Z}$ with respect to $\phi^{*}$, and taking account of the condition in (A13) in the Technical Appendix A. Thus the "normal" case is more likely to obtain when the average needle infection rate is high and a higher proportion of marginal IDUs are HIV-negative (that is $\frac{h}{j}$ is large). We also have the following result:

Result 8: For a sufficiently large $\pi$, normality obtains.

Proof: See Technical Appendix A.

The result implies that "normality" obtains when HIV prevalence rates are high. The intuition is as follows. When $\pi$ is very large, average needle infection is very high. Since average needle infection cannot be greater than one, reducing $\phi^{*}$ cannot increase it very much. (Stated differently, if $\alpha$ and $\omega$ are both large, shifting a few people between old and new needle use cannot change those two variables much). Thus the effect from fewer HIV-negative IDUs using old needles overwhelms any possible effect from greater average needle infection.

\section{Steady State Infections among Humans}

Thus far, we have taken the steady state rate of human infection $\pi$, as fixed. Here we make $\pi$ endogenous using a simple model of IDU population dynamics.

First, we hold Z (and hence $\phi^{*}$ and $\pi$ ) constant, and look for the steady-state population configuration. Let $\mathrm{N}$ denote the number of HIV-negative IDUs in the steady-state population and $\mathrm{S}$ the number of HIV-positive IDUs. Let $\mu$ denote the exogenously given rate at which HIVpositive IDU leave the population, whether through death, hospitalization, incarceration, or the cessation of injecting drug use. Then we have, 


$$
\dot{S}=\tau Z N-\mu S=\tau \lambda H N-\mu S
$$

In the steady state, we have $\dot{S}=0$ and $S=\frac{\tau Z}{\mu} N$. That is, in the steady state

$$
\pi=\frac{S}{S+N}=\frac{\tau Z}{\mu+\tau Z}
$$

Since $\pi=\frac{S}{S+N}$, we will have occasion to return to this steady state condition.

We assume that all IDUs who enter the population are HIV-negative and that entries occur at rate $\varepsilon$. It is easy enough to incorporate possibilities where this is not the case, as when people who become HIV-positive through sexual activity take to injecting drug use. Some HIV negative IDUs become HIV-positive and some cease being IDUs altogether. Let $\eta$ denote the rate at which HIV-negative IDUs leave drug injecting altogether. Hence

$$
\dot{N}=\varepsilon-\tau Z N-\eta N
$$

Setting $\dot{N}=0$, we obtain, in steady state

$$
\begin{aligned}
& N=\frac{\varepsilon}{\tau Z+\eta} . \\
& S=\frac{\varepsilon}{\mu} \frac{\tau Z}{\tau Z+\eta} \\
& N+S=\frac{\varepsilon}{\mu} \frac{\tau Z+\mu}{\tau Z+\eta}
\end{aligned}
$$

Thus, an NEP that reduced Z, ceteris paribus, would increase the number of HIVnegative IDUs and decrease the number of HIV-positive IDUs in steady state. The proportion of 
HIV-positive IDUs would, therefore, also fall. The effect on the total IDU population (N+S) depends on the relative sizes of the exit rates, $\eta$ and $\mu$. If $\mu>\eta$, that is, if HIV-positive IDUs exit injecting drug use at a rate faster than HIV-negative IDUs, then reducing $\mathrm{Z}$ increases the steady state population of IDU. If $\mu<\eta$, reducing $\mathrm{Z}$ decreases the steady state population. If death or hospitalization were the primary way that IDUs exited the population, then $\mu>\eta$ would be a safe assumption, but if decisions to abstain matter and HIV-negative IDUs are more optimistic and willing to invest, the opposite would hold. The net effect of these two forces is not immediately obvious.

The preceding analysis is incomplete, of course, because it holds constant $\pi$, the proportion of HIV-positive IDU as an argument of Z. We need a more careful analysis of how the equilibrium value of $\pi$ changes as $\phi^{*}$ changes. Using (19) and $\pi=\frac{S}{S+N}$, we can rewrite (19) as

$$
Z\left(\phi^{*}, \pi\right)=\frac{1}{\tau} \frac{\pi \mu}{1-\pi}
$$

Equation (22) describes the combination of $\mathrm{Z}$ and $\pi$ required for $\mathrm{S}$ and $\mathrm{N}$ to be in steady state. It is easy to confirm that $Z\left(\phi^{*}, 0\right)=0, Z\left(\phi^{*}, 1\right)=\infty, \frac{\partial Z}{\partial \pi}>0$ and $\frac{\partial^{2} Z}{\partial \pi^{2}}>0$. For future reference, also note that

$$
\left(\frac{\partial Z\left(\phi^{*}, \pi\right.}{\partial \pi}\right)_{\pi=0}=\frac{\mu}{\tau}
$$

On the other hand, recall from (16) that 


$$
Z\left(\phi^{*}, \pi\right)=H\left(\phi^{*}\right) \lambda\left(\phi^{*}, \pi\right)
$$

It can be readily checked that $Z\left(\phi^{*}, 0\right)=0$ and $Z\left(\phi^{*}, 1\right)=H\left(\phi^{*}\right)$ for all $\phi^{*}$. We also have

$$
\frac{\partial Z}{\partial \pi}=H\left(\phi^{*}\right) \frac{\partial \lambda}{\partial \pi}=H(1-H) g \frac{(1-J)}{q^{2}}\left(1-\frac{1-\pi}{q} \frac{g^{\prime}}{g} \frac{1-H}{1-J}\right)>0
$$

And, therefore, ${ }^{8}$

$$
\left(\frac{\partial Z\left(\phi^{*}, \pi\right.}{\partial \pi}\right)_{\pi=0}=\frac{H}{1-H}\left(\frac{3}{2}-J\right)
$$

Equations (22) and (24) for each $\phi^{*}$ determine the steady-state level of $\pi$. Multipleequilibria are a possibility. Note that the graphs traced out by the two equations always intersect at $\pi=Z=0$, i.e., there is always an equilibrium with no infection.

A necessary condition for the equilibrium with no infection to be unique is that the slope of (22) at the origin is greater than the slope of (24) (see Figure 1). That is

\section{INSERT FIGURE 1 ABOUT HERE}

$$
\frac{\mu}{\tau}>\frac{H}{1-H}\left(\frac{3}{2}-J\right)
$$

Thus, the no-infection equilibrium is likely to be unique if the rate of exits by HIVpositive IDUs is high, or the transmission rate $\tau$ is very low. The right hand side of (27) depends on $\phi^{*}$, but in a complicated way. It is possible that reducing $\phi^{*}$ could cause (27) to hold and thus

\footnotetext{
${ }^{8}$ To derive the result in (26) we have used the results from the technical appendix A that $\operatorname{Lim}_{x \rightarrow 0} g(x)=1, \operatorname{Lim}_{x \rightarrow 0} g^{\prime}(x)=-\frac{1}{2}$ from (A11), the fact that $q\left(\phi^{*}, 0\right)=1-H\left(\phi^{*}\right)$ and that $t\left(\phi^{*}\right) \omega\left(\phi^{*}\right)=0$ when $\pi=0$.
} 
all equilibria with infection to be eliminated, but the conditions under which this situation occurs are difficult to describe.

Only equilibria where the curve OB traced out by (22) cuts the curve OC traced by (24) from below are stable. Moreover, because OB tends to infinity as $\pi$ tends to unity, if there are any equilibria with infection at least one such equilibrium must be stable because (24) is bounded from above by one. A typical stable equilibrium is described by point $\mathrm{E}$ in Figure 2.

\section{INSERT FIGURE 2 ABOUT HERE}

Clearly, our major policy interest is in a stable equilibrium with infection. Suppose that we are in the "normal" case and that $\phi^{*}$ falls to $\bar{\phi}^{*}$. Then OC shifts downwards to OC' and the equilibrium moves from $\mathrm{E}$ to $\mathrm{E}^{\prime}$ : both $\mathrm{Z}$ and $\pi$ fall, as in Figure 3 .

\section{INSERT FIGURE 3 ABOUT HERE}

The decrease in $Z$, once infection adjusts to the new steady state is greater than the reduction we calculated in the previous section because we assumed $\pi$ to be unchanged.

Thus, all of the comparative steady-state results we derived in the first part of this section still hold when we allow $\pi$ to adjust as well as $\phi^{*}$. An effective NEP in the normal case reduces both the number and proportion of HIV-positive IDUs.

\section{Needle Supply}

In the normal case, reducing $\phi^{*}$ reduces infection among humans. The remaining question is whether NEPs reduce $\phi^{*}$. For this we need to return to the definition of $\phi^{*}$ given by 


$$
\phi^{*}=\frac{p_{n}-p-b(t)}{\lambda \tau}
$$

Thus, understanding the way needle prices $p_{n}-p$, are determined and in particular how NEPs affect them holds the key to understanding the influence of NEP on $\phi^{*}$.

We assume that needles are purchased in the market by needle rental firms that behave competitively. If q new needles per period are being acquired on the black market, their supply price is given by $n(q)$. The supply curve for needles in the black market is upward sloping $\left(n^{\prime}(q)>0\right)$. The easiest sources of needles are exploited first.

Needle rental firms maximize long-run average profit. In this section, we will assume that needle acquisition is their only cost. Once acquired, a needle lasts a maximum of $\mathrm{T}$ uses ( $\mathrm{T}$ sufficiently large), needs no maintenance, and imposes no storage or carrying costs on its firm. ${ }^{9}$ It is easy to relax this assumption; we shall discuss the implications of non-trivial carrying costs later.

As discussed in the section on individual choice, all users of needles can distinguish an unused (“new”) needle from a used (“old”) needle, but no user can distinguish among old needles of various ages. By contrast, needle rental firms can keep count and decide when to retire an older needle. Hence there are two rental prices: $\mathrm{p}_{\mathrm{n}}$ the rental price of new needles, and $\mathrm{p}$, the rental price of old needles.

These considerations allow us to describe the behavior of needle rental firms. Consider a firm that has decided to operate at the level of one needle per unit time. If we assume constantreturns-to-scale technology, this is an adequate description of all firms. At any given point in time in

\footnotetext{
${ }^{9}$ In practice, maintenance and storage for purposes of renting injecting equipment could impose significant costs on a needlerenting firm in the United States. For example, owners of shooting galleries, where needles are typically rented, could face up to 20 years' imprisonment and/or a \$500,000 fine under some provisions of federal law (Holden et al. 1991, p. 12). There are also state laws making it a crime to possess injecting equipment without a valid prescription.
} 
which the representative firm does not replace its needle, it earns a net profit of p. If it instead chose to replace its needle, it earns a net profit of $-n(q)+p_{n}$. Ruling out "corner" solutions, we have the following equilibrium condition:

$$
\begin{aligned}
& -n(q)+p_{n}=p, \text { or } \\
& p_{n}-p=n(q)
\end{aligned}
$$

It follows from (29) that the firm will be indifferent about when it replaces the needle. If the firm replaces the needle at age $t^{*}$, the zero-profit condition is

$$
n(q)=p_{n}+t^{*} p
$$

Comparing this expression with (29), we obtain the equilibrium rental prices

$$
\begin{aligned}
& p=0 \\
& p_{n}=n(q)
\end{aligned}
$$

In a model without maintenance, or carrying costs, the marginal cost of an additional injection is zero, so $\mathrm{p}=0$ should not be a surprising result. Incorporating these results in (28) we have that

$$
\phi^{*}=\frac{n(q)-b(t)}{\lambda \tau}
$$

Since average bluntness cost $b(t)$ is increasing in the number of uses "t", and moreover, from (8), " $\mathrm{t}$ " is decreasing in "q", we have that $-b(t)$ is increasing in "q". Since, by assumption the black market supply curve of new needles is upward sloping, we can write 


$$
\phi^{*}=\frac{R(q)}{\lambda \tau} \quad[R(q) \equiv n(q)-b(t)]
$$

Here $\partial \phi^{*} / \partial q$, showing that as the number of new needle users rises, the cost $\phi^{*}$ at which any IDU decides to move to using new needles also rises. We refer to (33) as the "supply curve” of new needle rentals, and depict it in Figure 4.

\section{INSERT FIGURE 4 ABOUT HERE}

\section{Needle markets}

For any given $\phi^{*}$, there is a "demand" for new needle rentals that is determined by the prevalence of HIV in the IDU population $\pi$ and the distributions H and J. Specifically, this demand is given by $q\left(\phi^{*}\right)$, where

$$
q\left(\phi^{*}\right)=\pi\left(1-J\left(\phi^{*}\right)\right)+(1-\pi)\left(1-H\left(\phi^{*}\right)\right)
$$

Call this the "demand function" of new needle rentals. Then equilibrium is determined by the intersection of supply and demand curves at point $\mathrm{F}$ in Figure 5.

\section{INSERT FIGURE 5 ABOUT HERE}

We assume that the intersection of the supply and demand curve defines an interior solution. If so, their respective slopes ensure that this needle market equilibrium is unique.

Note that in this section we are treating $\lambda$ and $\pi$ as exogenous: given $\lambda$ and $\pi$ we find the values of $q$ and $\phi^{*}$ that clear the needle market. But of course $\lambda$ and $\pi$ are endogenous, and the 
previous sections showed how they are determined from $q$ and $\phi^{*}$, resulting in an “interdependent” system, which is internally consistent.

\section{Needle Exchange Programs and Needle Legalization}

The policies that can be modeled intelligently in this setting are needle exchange and legalization of the sale of new needles. Why this should be the case with legalizing sale is obvious.

For needle exchange programs, the reasoning is not quite so obvious. Let $c$ denote the cost of exchanging a needle; this reflects time, the expected cost of arrest while traveling with a needle ${ }^{10}$ and possibly other factors. This cost establishes an upper bound to the black market price of new needles, equal to $c$. Then if a needle exchange does a fixed amount of exchanging, it is easy to see that the supply curve of new needle rentals looks like figure 6.A, with a flatter segment. Notice that it is still upward sloped due to fact that $-b(t)$ is increasing in "q". If the NEP does an unlimited amount of exchanging, the supply curve looks like figure 6.B

\section{INSERT FIGURES 6.A-6.B HERE}

The impact of lowering the supply curve can be seen in figure 7.

\section{INSERT FIGURE 7 ABOUT HERE}

As shown in Figure 7, lowering the supply curve of new needle rentals lowers $\phi^{*}$ and raises the proportion of new needle users among IDUs $q$. As $q$ rises, needle lifespan falls. The rise

\footnotetext{
${ }^{10}$ Even when needle exchange members are legally entitled to possess needles, regimes where it is illegal to possess and consume drugs typically result in the law enforcement machinery harassing NEP users who they suspect might also possess drugs on their person (personal observation by authors; Power 1988; Stimson 1988).
} 
in the number of new needles, however, is smaller than the number of new needles that the NEP gives out (if we are considering the supply shock as an NEP); thus there is some crowding out of other sources. Since needle cohorts become bigger, the steady-state rate of needle retirements must increase, too, but once again the increase in retirements is less than the number of retirements the NEP accomplishes. Needle retirements are crowded out, too.

All of these consequences rely on a lowering of the supply curve in the neighborhood of the equilibrium. If the supply curve were not lowered, the NEP would have no customers and hence would not affect anything; NEPs matter only if they lower price - just like sale legalization. Is it possible to have an NEP without the crowding out of needle creation and destruction as assumed, for instance in Kaplan (1989)? Yes, but only if the supply of black market needles were completely inelastic at the old equilibrium amount.

\section{General equilibrium}

The above needle market analysis is partial equilibrium because we hold the average needle infection rate $\lambda$ and the human infection rate $\pi$ constant. An NEP can reduce $\phi^{*}$ if the average needle infection rate does not change, but we know that reducing $\phi^{*}$ changes the average needle infection rate and the human infection rate.

First suppose that regularity and normality both obtain: reducing $\phi^{*}$ cuts both $\lambda$ and $\pi$. In the case of the demand curve, a lower $\pi$ shifts demand for new needles up: when more people are uninfected, the demand for new needles is greater. Ceteris paribus, such an upward shift in the demand for new needles raises $\phi^{*}$. In the case of the supply curve a lower $\lambda$ shifts the supply curve up: since old needles are less contaminated now, for any given supply price the person who is indifferent between old and new needles values his avoiding infection more highly. 
Ceteris paribus, such an upward shift in the supply curve for new needles raises $\phi^{*}$. Thus both general equilibrium effects raise $\phi^{*}$. But while these secondary effects reduce the size of the effect of NEPs, or of needle legalization, they cannot reverse it: because if they were to reverse it, there would be in equilibrium nothing to cause the changes in $\lambda$ and $\pi$ that resulted in the reversal.

If regularity does not obtain but normality obtains, then the secondary effects may increase the effect of an NEP, or they may not.

If neither regularity nor normality obtains, then the secondary effects magnify the effects of an NEP: they make the decrease in $\phi^{*}$ larger. But since normality does not obtain, we do not want to decrease $\phi^{*}$. In this case when NEPs are the most effective, we do not want to use them.

\section{Summary}

NEPs and needle legalization have two effects that unambiguously reduce steady-state human infection. First, they reduce $H\left(\phi^{*}\right)$, the proportion of HIV-negative IDUs using old needles. Second, they hasten the retirement of old needles. Both of these effects arise because they cut the cost of new needles.

On the other hand, lower prices for new needles also increase the proportion of HIV-positive IDUs using new needles. Sometimes, this will lead to a higher average infection rate of old needles. This perverse effect cannot occur if the expected number of times an old needle is used by an HIVpositive IDU exceeds 1.79, or if the distribution of subjective infection costs among HIV-positive IDU is fairly similar to the distribution among HIV-negative IDU, except for HIV-positive IDU who have no fear of infection. Even if this perverse effect occurs, it will be small if either the proportion of HIV-positive IDU or the proportion of old needles that are infected is large. 
When lower prices reduce the average needle infection rate, NEPs and needle sale legalization reduce human infection. When the average needle infection rate effect is small and in the opposite direction, the overall effect is the same.

\section{Carrying Costs of Injecting Equipment}

The model of the previous section can be extended in several directions. One obvious problem with the model is its neglecting the cost of holding a needle. This cost can be considerable, especially when possession of a needle is against the law (Holden 1991, Mahal 1995). Even in those jurisdictions where possession is legal, however, police may eye people with needles more suspiciously, so having a needle would raise the probability of arrest on other charges - possession of drugs, for instance.

Introducing "carrying costs" into the basic model is simple. Let $\sigma$ denote the per-period cost incurred by holding a needle. Thus a needle firm must incur $\sigma$ before the needle is used the first time, $2 \sigma$ before it is used the second time, and so on. Thus the zero profit condition becomes

$$
n(q)+t \sigma=p_{n}+(t-1) p
$$

The condition for supplier indifference between using needles only once and using them until they break stays the same because the carrying costs are incurred in each period.

$$
p_{n}-p=n(q)
$$

From (35) and (36), we have that

$$
p=\sigma
$$


From (36) and (37) we have

$$
p_{n}=n(q)+\sigma
$$

It follows from (38) that the supply curve in the new needle rental market is unchanged. The demand relationship stays the same, obviously. Needle life span and new needle demand stay the same, but IDUs become worse off. Thus other options, not discussed in this paper, might become attractive. Legalizing possession of needles is a way of reducing $\sigma$ and in the current model it has no public health consequences whatsoever.

\section{Drug Use}

The most common objection to NEPs and needle sale legalization proposals is that they may encourage drug use. In theory, NEPs could encourage drug use in two different ways: they could prolong the lives of IDUs by reducing infection, and they could encourage more people to become IDUs. We have already examined the first point with equation (21) on the steady-state number of drug users. Those opposed to NEPs probably do not object to increased survival of IDUs, and so we will not pursue this avenue further.

Accordingly, we consider changes in the population of IDUs due to changes in the rate of entry $\varepsilon$. Indeed, both the steady-state number of current IDUs (from (21)) and the number of individuals who become IDUs are proportional to $\varepsilon$. Thus, in order to consider the increased drug-use objection, we should make $\varepsilon$ endogenous. In general, the entry rate should depend negatively on the cost of injecting.

The cost of injecting for an individual with $\phi<\phi^{*}$ is $b(t)+\lambda \tau \phi$. NEPs reduce $b(t)$ and in the regular case reduce $\lambda$. For individuals with $\phi>\phi^{*}$ the cost of injecting is $n(q)$. An NEP also 
reduces this cost. Thus NEPs reduce the cost of injecting, especially in the regular case, and so should increase the entry rate $\varepsilon$.

But they may not increase it much. The cost of becoming an IDU is the cost of injectable drugs, the cost of possible incarceration, possible death (or severe injury) from overdose, or bad drugs, or drug-related violence, as well as the cost of injecting. Empirical studies have not found the elasticity of drug use with respect to the price of drugs to be great, and the cost of injecting is a small fraction of the cost of injectable drugs. For instance, Van Ours (1995) for opium in Indonesia during the Dutch colonial period, and Silverman and Spruill (1977) for heroin in Detroit, both find elasticities of demand with respect to price less than one. Saffer and Chaloupka (1995) and Chaloupka et al. (1998) find elasticities of participation for cocaine one of less than unity. Grossman et al. (1996) use the rational addiction framework of Becker and Murphy (1988) and found short run elasticities of cocaine use of 0.7 and a long-run elasticity of 1.2 among American youth. In line with this literature, suppose that the elasticity with respect to the price of injectable drugs is one. If the cost of injecting is about a fifth of the cost of drugs (\$1 for a \$5 bag), then the elasticity of use with respect to the price of injecting is 0.2 .

Of course, theory thus does not lead us to believe that a stand-alone NEP would reduce entry into drug use. On the contrary, NEPs by themselves probably increase entry into drug use. (By the same argument, the availability of treatment probably increases entry into drug use, because it makes exit from addiction easier). But the effect may be small enough so the ancillary services provided by NEPs are powerful enough to offset any effect on entry.

\section{Simulation Results}

In this section we present simulation results for the epidemiological framework used in this paper under several different scenarios. The simulation results are based on the "Lump Difference" 
case for the distribution of costs of infection with HIV, with the further assumption that $H(\phi)$, the distribution of the cost of infection to an HIV-negative person is of the one-parameter exponential variety. The expected value of the cost of HIV infection to an HIV-negative individual $E(\phi)$ was taken to be identical to the discounted monetary value of statistical life years forgone for an average HIV-negative IDU infected today. We concentrate on the parameters that might be observed in developed countries.

Because $E(\phi)$ is also the inverse of the exponential density parameter $\rho$, information on the monetary value of life years lost owing to HIV infection enabled us to estimate the parameter of the exponential density, namely $\rho$. An estimate of US\$460 thousand was used for the value of life years lost by an HIV-negative IDU to an HIV-infection. The precise details of the method used to calculate the value of life years lost are described in Technical Appendix C, and we provide only an outline here. First, studies on long-term survival among IDUs were used to construct life tables, with and without HIV infection, for IDUs. These yield the conclusion that in the US and EU an IDU aged 20 years who acquires an HIV infection, can expect to live anywhere between 22.1 years and 35.5 years less than if he were not HIV-infected. Second, estimates of incomes of drug users and prevailing unemployment rates among IDUs were used to assess that the average incomes of IDUs were at most half that of non-drug users. Third, estimates of a statistical life and income elasticity of the value of life from Viscusi and Aldy (2003) were used to estimate the value of life of an IDU to be of the order of US\$4.35 million. Discount rates reported in Bretteville-Johnson (1999) and Kirby and Petry (2004) and life expectancy at age 20 for an HIV-negative IDU were used to estimate the (annualized) value of a statistical life year. Finally, life tables for HIV-negative and HIV-positive IDU were combined with information on discount rates and the value of a statistical life year were used to the value of life years foregone due to HIV-infection. 
Four different values of $\mathrm{L}$, the proportion of HIV-positive IDU who are tested were considered: 5\%, 20\%, 40\% and 60\%. Even higher proportions of HIV-positive individuals being tested were ruled out owing to the considerable stigma associated with drug use, the underground nature of the drug economy, and so forth. We assumed zero bluntness costs of using needles and zero carrying costs. The transmission rate of HIV from an infected needle to an HIV-negative IDU was taken to be $1 / 300$. Bleach use rates were taken to range from $20 \%$ to $80 \%$ ( $80 \%$ was the rate considered in Kaplan and O’Keefe (1993)).

Next we needed (exogenously determined) estimates of entry and exit rates of HIV-negative and HIV-positive persons into (and out of) the IDUs population. There are three main ways of exiting the IDU population: death, incarceration, or cessation of injecting drug use (Note that exit via HIV-infection of an HIV-negative IDU is separately accounted for in our model). Of course, the death rates among HIV-positive IDUs would be higher than death rates among HIV-negative IDUs. Using studies that examined cessation rates of injection drug use among IDUs populations in the United States and Europe, we assumed an annual cessation rate of 5 percent per year. Life tables for HIV-negative IDUs and HIV-positive IDUs were used to arrive at estimates of annual mortality rates of $1 \%$ and $5 \%$ in the two groups, respectively. In general, in the United States, about $11 \%$ $14 \%$ of IDUs get arrested each year, with the average length of incarceration a little more than one year, amounting to an annual exit rate of 11\%-14\%, for HIV-negative and HIV-positive IDUs. Assuming, however, that release from prison and entry is uniformly distributed throughout the year and that, upon release, the former prisoners re-enter the IDU population, imprisonment would have no effect on exit rates. Assuming statistical independence across reasons for exit, we have that the annual exit rate among HIV-negative IDUs is 5.95\% and among HIV-positive IDUs, 9.75\%. These 
annual rates were converted into “daily exit rates” of 0.000163 for HIV-negative IDUs (the parameter $\eta$ ) and 0.000267 for HIV-positive IDUs (the parameter $\mu$ ) .

The entry rate for HIV-negative IDUs into the population (the parameter $\varepsilon$ ) was estimated as follows. Using estimates of IDU population of 1.3 million in the United States and HIV infection rates among IDUs reported in Aceijas et al. (2004) and the exit rates estimated above, we calculated the total annual number of exits from HIV-negative IDUs (excluding conversion to HIV-positive status) and HIV-positive IDUs in the USA to be 62,400 and 26,000, respectively. Assuming these IDU populations to be in steady state and the only source of (net) entry into the HIV-positive population to be via HIV-infection acquired by an HIV-negative drug user, yields an entry rate of HIV-negative IDU of 88,400, or a daily average of 242.

Finally, we considered a specific functional form (constant elasticity) of the supply function of new needles $n(q)$, pre-NEP, given by

$$
q=K P_{n}^{\delta}
$$

Here $P_{n}$ is the price of a new needle, $\mathrm{K}$ is a scale parameter and $\delta$ is a parameter indicating the elasticity of the new needle supply curve. We experimented with four values of $\delta$ in our simulation exercises, ranging from 0 to 1 , indicating differing levels of responsiveness of new needle supplies in the market. In the first set of set of simulations, the parameter $\mathrm{K}$ was taken to be 100,000, calibrated to roughly equal 10 uses per needle in the situation when the elasticity of the supply curve of new needles is zero (the supply setting most similar to that of the Kaplan-O’Keefe (1993) model). 
The size E, of a Needle Exchange Programs (NEP) was taken to denote the proportion of new needles in circulation at any given time accounted for by the program. Four values of $\mathrm{E}$ were considered, $0,0.25,0.50$ and 0.75 , in increasing order of scale of the needle exchange programs.

Table 1 describes the parameter estimates used our simulation exercises. The simulation results themselves are provided in Tables 2 through 8 . Tables 2 to 4 present simulation results that describe, for bleach use rates of $20 \%, 50 \%$, and $80 \%$, respectively, and a proportion L of HIVpositive individuals who know their status, of $20 \%$, how increases in the size of a needle exchange program (E) are related to steady-state levels of infections in needles (the average rate $\lambda$ and the terminal rate $I$ ) and humans ( $\pi$ ), the proportion of users of new needles who are HIV-positive $(\alpha)$, the proportion of users of old needles who are HIV-positive $(\omega)$, the average number of numbers a needle is used $(1+t)$, and the cut-off value of the $\operatorname{cost} \phi^{*}$ above which individuals choose a new needle to inject. The results in Tables 2 through 4 indicate that progressive increases in the size of the needle exchange program lower the steady-state values of all of the above variables. For instance, in a setting where the elasticity of new needle supply is zero, an increase in the size of $\mathrm{E}$ from zero to a level where it supplies $75 \%$ of new needles used by IDU, reduces, in steady state, the average number of times a needle is used from 10.64 to 2.92. Steady-state infection in humans falls from $58 \%$ to about $39 \%$; the average needle infection rate from $12.6 \%$ to $8.2 \%$ and the terminal needle infection rate from $64 \%$ to $45 \%$. The results show, not surprisingly, that increases in the supply elasticity of new needles result in lower initial needle and population infection rates.

In our simulations, average and terminal needle infection rates, serve as the upper and lower bounds of population infection rates. Notice, however, the closeness of terminal needle infection rates and HIV-prevalence in the IDU population, thereby suggesting that data on the former could be used as a proxy for the latter if HIV testing is not feasible in such populations. Tables 2 through 4 
also indicate, for a given proportion of HIV+ population that knows its infection status (20\%), that lowered bleach use rates bring the average and the terminal needle infection rates closer, raise steady-state population infection rates, and lower the average number of times a needle is used.

Tables 5 through 8 explore the likely impact of changes in the proportion of $\mathrm{HIV+}$ individuals tested for HIV, for a fixed bleach use rate, taken for our purposes, to be $50 \%$. As a practical matter this can occur if testing services are expanded, particularly as part of treatment programs. Our results show, first, that even for large changes in the proportion tested - ranging from $5 \%$ to $60 \%$, the steady-state impact on needle and human HIV-infection rates is not particularly large. However, the testing of a greater proportion of HIV-positive individuals gives rise to a sorting effect, whereby the proportion of older needles being used by HIV-positive individuals ( $\omega$ ) rises relative to the proportion $(\alpha)$ of new needles being used by them. This, in turn has the effect of lowering steady state HIV-infections in the IDU population.

Note from Tables 2 through 8 that one of our key partial equilibrium results do not hold when we consider steady-state outcomes: namely, the impact of increased NEP size on the proportion of users of new and old needles who are HIV-positive, $\alpha$ and $\omega$, respectively (Result 2). The expansion of an NEP does reduce the cut-off cost of being infected with $\operatorname{HIV} \phi^{*}$, as noted earlier, but it is accompanied by reductions in steady state values of both $\alpha$ and $\omega$ - that is, the short-run (immediate) effect of an NEP and its long-run effect move in opposite directions.

In Tables 9 and 10 we compare the results from our behavioral model with those of the mathematical model used by Kaplan-O'Keefe (1993) in their seminal paper (for details on the version of the Kaplan-O’Keefe model used in this paper, see Technical Appendix D). In Table 9, we compare the two models using a subset of parameter values from Table 1. Specifically, the daily exit rate of HIV+ individuals was taken to be 0.000267 , the bleach use parameter to be 0.80 , the rate 
of transmission from an infected needle to an uninfected human to be $1 / 300$, the supply function scale parameter of 100,000 and a ratio of shared needles to IDU population equal to unity, since every IDU injects once in every period in our model. However, in undertaking this comparison there is a small complication that needs to be addressed. Because the NEP size parameter $(\kappa)$ in the Kaplan and O'Keefe model is a proportion that indicates the number of "used" needles that are taken out of the system at any given time, whereas the NEP size parameter in our model (E) is the proportion of new needles supplied by an NEP, we need to express $\mathrm{E}$ in terms of $\kappa$. By definition, $\kappa$ times the number of used needles must equal $\mathrm{E}$ times the number of new needles. Thus, we have that, in steady state

$$
\kappa=\frac{E^{*} \text { new }}{\text { used }}=\frac{E q^{*}}{(1+t) q^{*}}=\frac{E}{(1+t)}
$$

Using (40) in conjunction with the above mentioned parameter values we can generate a set of results on HIV-infection in needles and humans in the Kaplan-O'Keefe model that are comparable to our behavioral model. These results are provided in Table 9. The case that is most relevant for comparison, given that in the Kaplan-O'Keefe model needles live forever, is the one with new needle supply elasticity $\delta$ of zero. We find that although both models predict declines in HIV-prevalence among humans and people after the introduction of an NEP, the decline is much greater in the behavioral model, for any given size of the NEP. For cases with supply elasticity greater than zero, using the Kaplan-O’Keefe would yield increasingly inaccurate estimates of the pre- and post-NEP IDU infection rates, if HIV transmission among IDUs is along the lines described in this paper, and not by a model where needles and humans were randomly matched. In 
general, estimates based on the Kaplan model would upwardly bias assessments of baseline HIVprevalence (prior to the setting up of an NEP) and average needle infection rates by substantial margins.

Note also that the needle infection rates in the two models are quite different. The KaplanO'Keefe model predicts much higher average needle infection rates $(\bar{n})$ in each steady state compared to the average needle infection rates predicted by the behavioral model $\lambda$. In fact, the estimated needle infection rates under the Kaplan-O’Keefe model would even exceed substantially the terminal needle infection rates based on the behavioral model.

\section{Inferences about human infection}

The final question we examine is whether the inferences that based on the Kaplan and O’Keefe model regarding the relationship between terminal needle infection rates in New Haven to changes in human infection are reasonable. To do this, we constructed examples where terminal needle infection in the absence of an NEP was 67 percent—-the Kaplan-O'Keefe observation in their New Haven study. We then found the size of NEP that would be required to lower the terminal needle infection rate to the 40 percent observed by them, post-NEP. This calculation was undertaken under different assumptions about needle supply elasticity. ${ }^{11}$

The corresponding human infection rates are reported in Table 10, along with the NEP sizes required for the purpose. The results in Table 10 suggest that the results based on the KaplanO’Keefe model are not robust. In general, the decrease in human infection that accompanies this decrease in terminal needle infection is larger in our model than it is in Kaplan-O'Keefe model. Usually the relative size of the NEP is considerably different from the relative size of the NEP that

\footnotetext{
${ }^{11}$ For this purpose we calibrated the scale parameter $(\mathrm{K})$ for the needle supply function. For further details see note to Table 10.
} 
Kaplan and O'Keefe believed they were observing in New Haven. (They knew for certain the absolute size of the NEP, but without knowing the flow of needles from other sources they did not know the relative size, and the relative size is what is relevant.). Our findings suggest that one must know must more about the environment in which an NEP is operating in order to know its likely effects on human infection.

\section{Discussion and Concluding Remarks}

We have shown that needle use is amenable to analysis by economic techniques. On a theoretical level, our major conclusions are the (consequential) equivalence of needle exchange programs and legalization of needle sales, and the vastly different consequences of legalizing sale and legalizing possession. Our analysis also suggests -- although much more empirical work is needed on this issue -- that the estimates in the epidemiological literature of the impact of NEPs and sale legalization on the spread of AIDS are not overstated. If anything, rational behavioral responses probably magnify the impact of these interventions, rather than offset it - both because of lowered needle circulation times and because of sorting effects in the IDU population. Our theoretical and simulation results confirm the finding from the literature that NEPs lead to a decline in needle sharing behavior, and confirm other correlates of NEP, such as reducing sharing and reductions in the average number of times a needle is used (DeSimone 2002a; Broadhead et al. 2000; Sears et al. 2001).

Much work remains to be done on this topic. Technical matters, such as the possibility of super-infection, and the varying transmission rates of different strains of HIV are one obvious concern. As long as the cost of re-infection is lower than the cost of initial infection, neither is crucial to our analysis. Obviously, a broader range of numerical estimates of the parameters in these 
models, particularly in developing and high-risk countries, need to be made. The cost-effectiveness of NEPs ought to be assessed under alternative assumptions about the elasticity of needle supply.

Theoretical issues, too, need further exploration. We need to better account for sexual transmission of HIV among IDU. Another key issue is imperfect competition in needle markets. Entry into the needle-selling market appears to be easy - diabetics obtain many needles, and getting wholesalers to make bulk shipments into proscribing states does not appear to be difficult (Hopkins 1988). But search and transactions costs for IDUs are very high, primarily because drug use and possession are illegal activities. Sellers, then, may have considerable market power in individual transactions - which is why the law of one price apparently does not hold in this market. Since sellers will still have zero expected profit, a model with seller market power will probably not alter the fundamental conclusions in this paper, but it merits investigation. In our model, unemployment was introduced in the calculation of losses due to HIV infection - by reducing this loss, unemployment is likely to increase the likelihood of an IDU using an older needle. Unemployment may, however, have other implications - including the likelihood of crime (and associated prison stays). It may also lead to formation of needle/drug sharing groups as IDUs seek to reduce the risk of facing withdrawal symptoms. This too, it appears to us, is an important area for future work.

Although the paper does do so, the needle market should ideally not be decoupled from the drug market. On the one hand, drug market reactions could offset some of the public health benefits of needle supply-side interventions. Needle price decreases raise the demand for drugs (since needles and drugs are complements), so if drug supply is less than perfectly elastic, they raise the equilibrium price of drugs. Higher drug prices show up in decreased drug purity (since transactions costs demand that drugs be sold only in $\$ 5, \$ 10$, and \$20 units). Decreased drug purity encourages the spread of AIDS, partly because it is likely to increase the number of injections per week that 
IDUs require. An attention to drug markets and drug policy could potentially better align our model to the common observation of needle sharing in peer groups that may form, for instance, to address the risk of drug overdoses, or ensuring a regular supply of drugs. 


\section{References}

Aceijas, Carmen, Gerry Stimson, Matthew Hickman, Tim Rhodes. 2004. "Global overview of injecting drug use and HIV infection among injecting drug users” AIDS 18(17):2295-303.

Anderson, Warwick. 1991. "The New York Needle Trial: The Politics of Public Health in the Age of AIDS" American Journal of Public Health 81(11):1506-17.

Becker, Gary, Kevin Murphy. 1988. "A theory of rational addiction” Journal of Political Economy 96(4):675-700

Blower, Sally, D. Hartel, H. Dowlatabadi, R. Anderson, R. May. 1991. "Drugs, sex and HIV: a mathematical model for New York City.” Philosophical Transactions of the Royal Society London, Series B (Biological Sciences) 331:171-87.

Bloom, David, A. Mahal and D O'Flaherty "Economic perspectives on injecting drug use” Advances in Health Economics and Health Services Research 16:371-95, 2005

Brettle, R., A. McNeil, S.Burns, S. Gore, A. Bird, P. Yap, L. MacCallum, C. Leen, A. Richardson. 1996. "Progression of HIV: follow-up of Edinburgh injecting drug users with narrow sero-conversion intervals in 1983-85” AIDS 10(4): 419-30.

Bretteville-Jensen, A.L. 1999. "Addiction and discounting” Journal of Health Economics 18:393-407.

Broadhead, Robert, Yael van Hulst, Douglas Heckathorn. 2000. "The impact of a Needle Exchange’s Closure,” unpublished. New Haven, CT: Yale University, Department of Sociology.

Center for Drug Use and HIV Research (CDUHR) News. 2005. "Medical conditions related to high-risk drug use” Fall/Winter 2005/6, 8(2):1-2 
Center for Research on Environment, Health and Population Activities. 2002. Injecting and sexual behaviors of male injecting drug users in Kathmandu valley. Kathmandu, Nepal: Family Health International.

Chaloupka, Frank, Michael Grossman, J. Tauras. 1998. "The demand for cocaine and marijuana by youth.” Working paper no. 6411. Cambridge, MA: National Bureau of Economic Research.

Chitwood, D., M. Comerford, K. Kitner, W. Palacios, J. Sanchez. 2001. “A comparison of HIV risk behaviors between new and long-term injection drug users" Substance Use and Misuse 36(12):91-111.

De Angelis, Daniela, Matthew Hickman, Shuying Yang. 2004. "Estimating long-term trends in the incidence and prevalence of opiate use/injecting drug use and the number of former users: Back calculation methods and opiate overdose deaths." American Journal of Epidemiology 160(10):994-1004

Des Jarlais, Don, Samuel Friedman. 1994. "AIDS and the Use of Injected Drugs” Scientific American 270(2): 82-8.

Desenclos, Jean-Claude, George Papaevangelou, Rosemary Ancelle-Park. 1993. "Knowledge of HIV Serostatus and Preventive Behavior among European Injecting Drug Users” AIDS 7(10):1371-7.

DeSimone, Jeff. 2002a. "Determinants of drug injection behavior: Economic factors, HIV injection risk and needle exchange programs,” working Paper \#9350. Cambridge, MA: National Bureau of Economic Research.

DeSimone, Jeff. 2002b. "Illegal drug use and employment” Journal of Labor Economics 20(4):952-77. 
Doherty, M, R Garfein, E Monterroso, D Brown, D Vlahov. 2000. “Correlates of HIV infection among young adult short-term injection drug users” AIDS 14:717-26.

Estrada, A. 1998. "Drug use and HIV risks among African-American, Mexican-American and Puerto Rican drug injectors” Journal of Psychoactive drugs 30(3):247-53.

Michael French, Gary Zarkin, Laura Dunlap. 1998. "Illicit drug use, absenteeism and earnings at six U.S. worksites” Contemporary Economic Policy 16(3): 334-46.

N. Galai, M. Safaeian, D. Vlahov, A. Bolotin and D. Celentano. 2003. "Longitudinal patterns of drug injection behavior in the ALIVE study cohort, 1988-2000: Descriptions and Determinants” American Journal of Epidemiology 158(7):695-704.

Ghodse, Hamid, Adenekan Oyefeso, Bridget Kilpatrick. 1998. "Mortality of drug addicts in the United Kingdom 1967-93” International Journal of Epidemiology 27:473-8.

Grossman, Michael, Frank Chaloupka and C. Brown. 1996. "The demand for cocaine by young adults: A rational addiction approach.” Working paper no. 5713. Cambridge MA: National Bureau of Economic Research.

Holden, Gwen, Penny Wakefield, Richard Rogers, Andrew Waghorn, Michelle Lehmann. 1991. A Guide to State Controlled Substances Acts. Washington, D.C.: National Criminal Justice Association.

Hser, Yih-Ing, Valerie Hoffman, Christine Grella, and Douglas Anglin. 2001. “A 33-year followup of narcotics addicts” Archives of General Psychiatry 58(May): 503-8.

Hopkins, William. 1988. "Needle Sharing and Street Behavior in Response to AIDS in New York City." In R. Battjes and R. Pickens (eds.) Needle Sharing Among Intravenous Drug Abusers: National and International Perspectives. NIDA Research Monograph No. 80. Washington, D.C.: Public Health Service. 
Hurley, S. F., D. J. Jolly, J.M. Kaldor. 1997. "Effectiveness of needle-exchange programmes for prevention of HIV infection” Lancet 349 (9068):1797-1800.

Kaplan, Edward. 1989. "Needles that Kill: Modeling Human Immunodeficiency Virus Transmission via Shared Drug Injection Equipment in Shooting Galleries" Reviews of Infectious Diseases 11(2):289-98.

Kaplan, Edward, and Elaine O'Keefe. 1993. "Let the Needles do the Talking! Evaluating the New Haven Needle Exchange" Interfaces 23(1):7-26.

Kirby, K.N., N.M. Petry. 2004. "Heroin and cocaine abusers have higher discount rates for delayed rewards than alcoholics or non-drug using controls” Addiction 99(4):461-71.

Kniesner, Thomas, W. Kip Viscusi, Christopher Woock, James Ziliak. 2005. "How unobservable productivity biases the value of a statistical life.” Discussion paper no. 524. Cambridge MA: Harvard Law School.

Kremer, Michael. 1996. "Integrating Behavioral Choice into Epidemiological Models of AIDS" Quarterly Journal of Economics May: 549-73.

Law, M., M. Lynskey, J. Ross et al. 2001. “Back projection estimates of the number of dependent heroin users in Australia” Addiction 96:433-43.

Leonard, Lynne, Leslie Forrester, Christine Navarro, Janice Hansen, and Cynthia Doucet. 1999. The Effective of Needle Exchange Programs in Modifying HIV-Related Outcomes: A Systematic Review of the Evidence 1997-1999. Ottawa: University of Ottawa, Department of Epidemiology and Community Medicine, Community Health Research Unit. 
Lloyd-Smith, Elisa, Elizabeth Brodkin, Evan Wood, Thomas Kerr, Mark Tyndall, Julio Montaner and Robert Hogg. 2006. Impact of HAART and injection drug use on life expectancy of two HIV-positive cohorts in British Columbia” AIDS 20:445-50.

Lurie, Peter, Arthur Reingold, Benjamin Bowser, Donna Chen, Jill Foley, Joseph Guydish, James Kahn, Sandra Lane, and James Sorensen. 1993. The Public Health Impact of Needle Exchange Programs in the United States and Abroad. Berkeley, CA: University of California, School of Public Health.

MacDonald, M., M. Law, J. Kaldor, J. Hales, G. Dore. 2003. "Effectiveness of needle and syringe programmes for preventing HIV transmission,” International Journal of Drug Policy 14(5-6): 353-357.

Magura, Stephen, Joel Grossman, Douglas Lipton, Qudsia Siddiqi, Janet Shapiro, Ira Marion, Kenneth Amann. 1989. "Determinants of Needle Sharing Among Intravenous Drug Users" American Journal of Public Health 79(4):459-62.

Mahal, Ajay. 1995. Essays on the Economics of the HIV/AIDS Epidemic. New York: Columbia University, Department of Economics.

Mandell, W., J. Kim, C. Latkin, T. Suh. 1999. "Depressive symptoms, drug networks and their synergistic effects on needle sharing behavior among street injection drug users” American Journal of Alcohol and Drug Abuse 25(1):117-27.

Murray, John, Matthew Law, Zhanhai Gao, John Kaldor. 2003. "The impact of behavioral changes on the prevalence of human immunodeficiency virus and hepatitis $\mathrm{C}$ among injection drug users.” International Journal of Epidemiology 32:708-14.

Nadelmann, Ethan, Peter Cohen, Ernest Drucker, Ueli Locher, Gerry Stimson, and Alex Wodak. 1994. "The Harm Reduction Approach to Drug Control: International Progress.” Unpublished. Princeton, NJ: Princeton University, Woodrow Wilson School of Public Affairs. 
Philipson, Tomas, Richard Posner. 1993. Private Choice and Public Health: The AIDS Epidemic in an Economic Perspective. Cambridge, MA: Harvard University Press.

Pollack, H. 2001. "Cost-effectiveness of harm reduction in preventing Hepatitis C among injection drug users” Medical Decision Making 21:357-67.

Power, Robert. 1988. "The Influence of AIDS Upon Patterns of Intravenous Use--Syringe and Needle Sharing--Among Illicit Drug Users in Britain." In R. Battjes and R. Pickens (eds.) Needle Sharing Among Intravenous Drug Abusers: National and International Perspectives NIDA Research Monograph \#80. Washington, D.C.: Public Health Service.

Robertson, J., P. Ronald, G. Raab, A. Ross and T. Parpia. 1994. "Deaths, HIV infection, abstinence, and other outcomes in a cohort of injecting drug users followed up for 10 years,” British Medical Journal 309:369-70.

Saffer, Henry, Frank Chaloupka. 1995. “The demand for illicit drugs,” National Bureau of Economic Research working paper no. 5238. Cambridge, MA: NBER.

Sears, Clare, Erin Weltzien, Joseph Guydish. 2001. “A cohort study of syringe exchangers and non-exchangers in San Francisco” Journal of Drug Issues 31(2): 445-64.

Silverman, L. P., N.L. Spruill. 1977. "Urban crime and the price of heroin” Journal of Urban Economics 4(1): 80-103.

Stimson, Gerry. 1988. “Injecting Equipment Exchange Schemes in England and Scotland.” In R. Battjes and R. Pickens (eds.) Needle Sharing Among Intravenous Drug Abusers: National and International Perspectives NIDA Research Monograph \#80. Washington, D.C.: Public Health Service 
Termorshuizen, Fabian, Anneke Krol, Maria Prins, Erik van Ameijden. 2005. "Long-term outcome of chronic drug use: The Amsterdam cohort study (ACS) among drug users,” American Journal of Epidemiology 161(3):271-9.

Terza, Joseph, Peter Vechnak. 2001. "The effect of substance abuse on employment status.” University Park, PA: Pennsylvania State University, Department of Economics.

Tilson, H., A. Aramrattana, S. Bozzette, D. Celentano, M. Falco, T. Hammett, A. Kozlov, S. Lai, A. Mahal, R. Schottenfeld, S. Solomon. 2007. Preventing HIV transmission among injecting drug users in high risk countries. Washington, D.C.: National Academies Press.

Tsui, Rose, Belinda Herring, Jason Barbour, Robert Grant, Peter Bacchetti, Alex Kral, Brian Edlin, Eric Delwart. 2004. "Human Immunodeficiency Virus Type 1 Super-infection was not detected following 215 years of Injection Drug User exposure.” Journal of Virology 78(1):94103.

Turner, C., H. Miller, L. Moses (editors). 1989. AIDS: Sexual Behavior and Intravenous Drug Use. Washington, D.C.: National Academy Press.

UNAIDS. 2008. Report on the Global AIDS Epidemic. Geneva: UNAIDS.

[http://data.unaids.org/pub/GlobalReport/2008/JC1511_GR08_ExecutiveSummary_en.pdf]

UNAIDS. 2007. Latin America: AIDS epidemic update. Geneva: UNAIDS.

[http://data.unaids.org/pub/Report/2008/jc1530_epibriefs_latinamerica_en.pdf]

van Asten, Liselotte, Faroudy Boufassa, Veronique Schiffer, Raymond Brettle, J. Roy Robertson, Ildefonso Aguado, Jim McMenamin, Robert Zangerle, Arnaud Fontanet, Roel Coutinho, Maria Prins. 2003. "Limited effect of highly active antiretroviral therapy among HIV-positive injecting drug users on the population level” European Journal of Public Health 13(4):347-9. 
van der Kuyl, Antoinette, Marion Cornelissen. 2007. "Identifying HIV-1 dual infections"

Retrovirology 4:67

Van Ours, J. 1995. "The price elasticity of hard drugs: the case of opium in the Dutch East Indies, 1923-1938” Journal of Political Economy 103: 261-279.

Vickerman, Peter, Charlotte Watts. 2003. "Injecting drug use and the sexual transmission of HIV: simple model insights” International Journal of Drug Policy 14:89-93.

Vickerman, Peter, M Hickman, T Rhodes, Charlotte Watts. 2006. "Model projections on the required coverage of syringe distribution to prevent HIV epidemics among injecting drug users” Journal of Acquired Immune Deficiency Syndromes 42(3):355-61.

Vickerman, Peter, M Hickman, A Judd. 2007. "Modeling the impact on Hepatitis C transmission of reducing syringe sharing: London case study.” International Journal of Epidemiology

Viscusi, W. Kip, Joseph Aldy. 2003. "The value of a statistical life: A critical review of market estimates throughout the world” Working paper no. 9487. Cambridge MA: National Bureau of Economic Research.

Williams, M., Z. Zhao, A. Bowen, R. Freeman, W. Elwood, R. Rusek. 1997. "Introduction of HIV into drug injector networks outside AIDS epicenters” International Journal of STD and AIDS 8:629-35.

Zarkin, Gary, Thomas Mroz, Jeremy Bray, Michael French. 1998. "The relationship between drug use and labor supply for young men” Labour Economics 5(4): 385-409. 
FIGURE 1: Unique Equilibrium at $(0,0)$

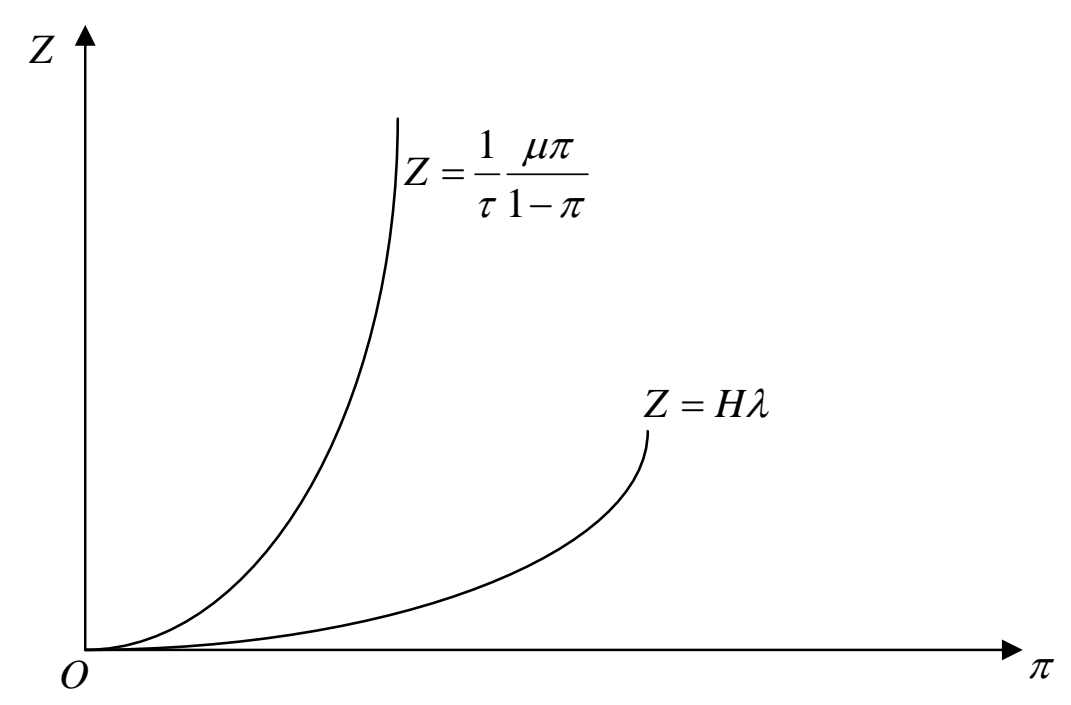

FIGURE 2: Stable $\left(Z, \phi^{*}\right)$ Equilibrium

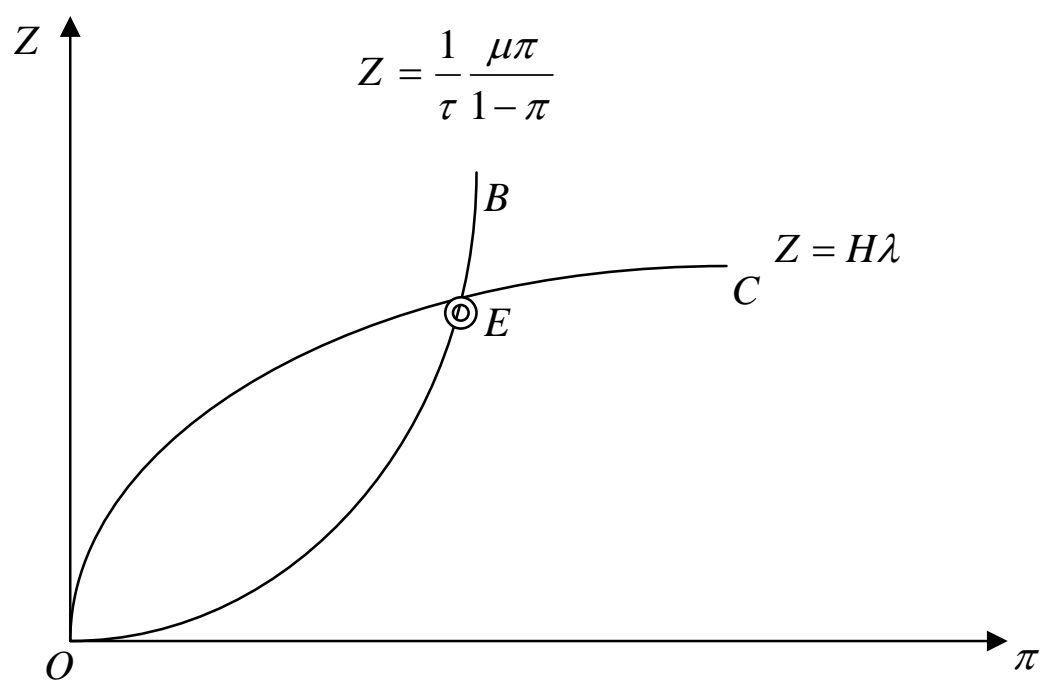


FIGURE 3: Partial Equilibrium Effect of fall in $\phi^{*}$ on Stable Steady State Equilibrium

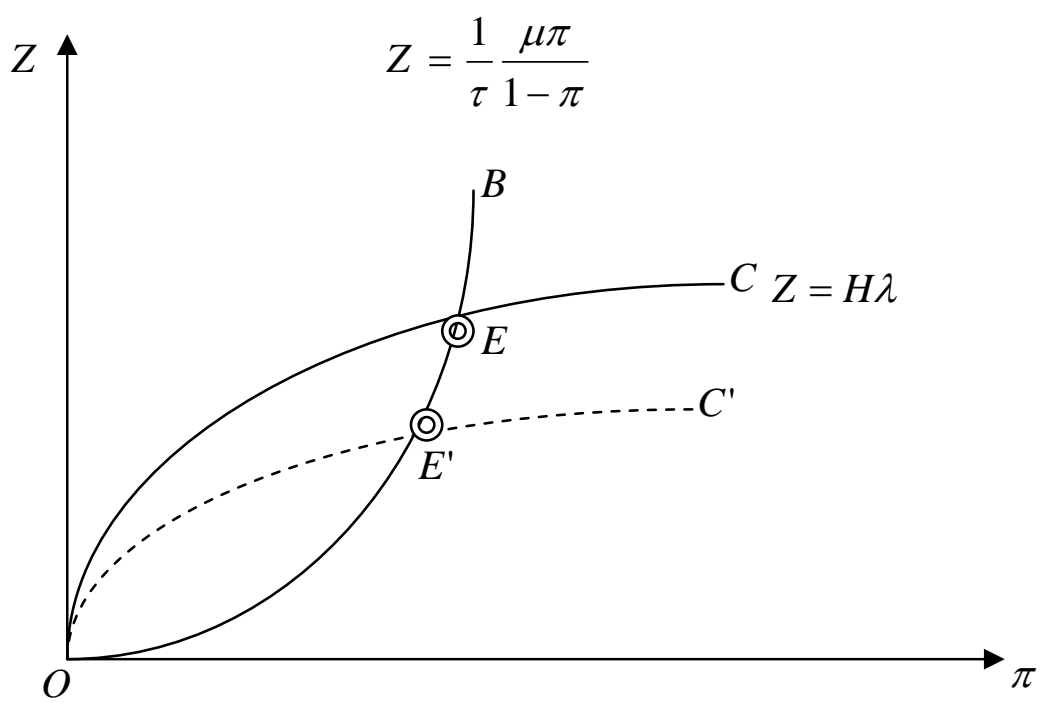

FIGURE 4: The "Supply Curve” for New Needle Rentals

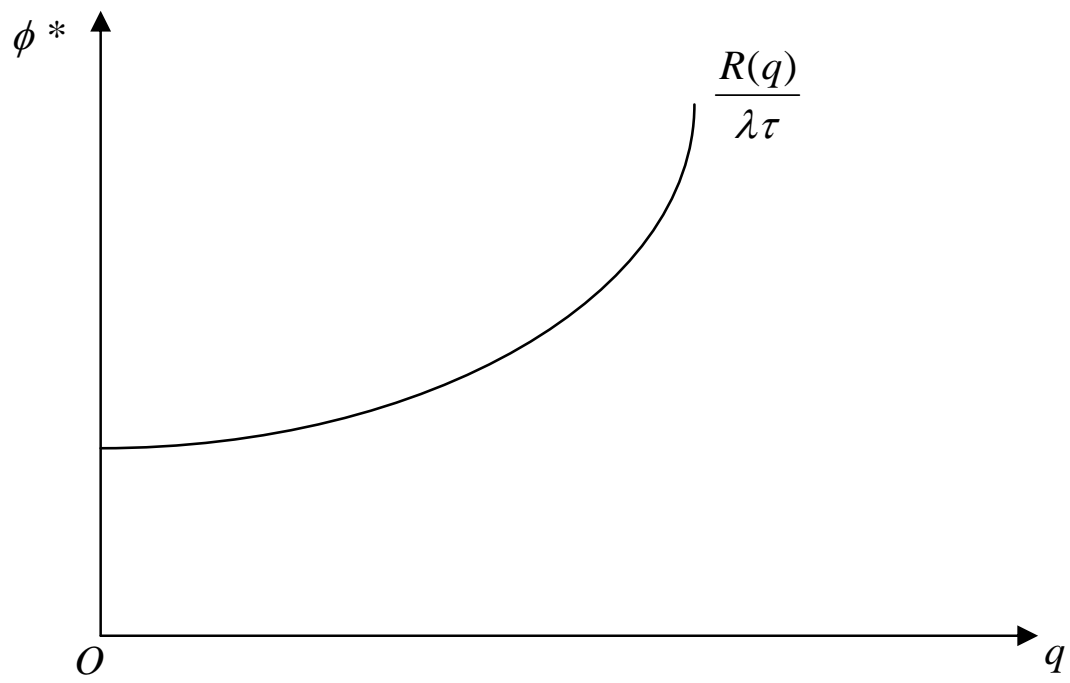


FIGURE 5: Equilibrium in the New Needle Rental Market

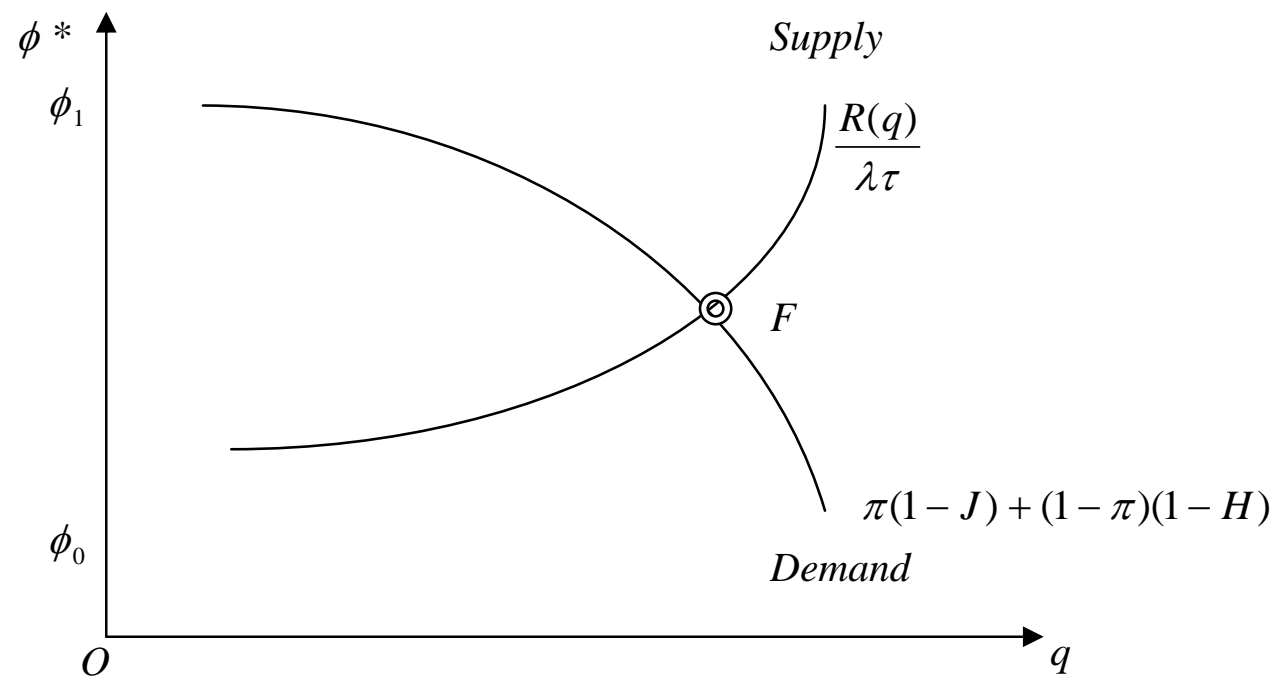

FIGURE6.A: New Needle Rental Supply Curve with Limited Size NEP

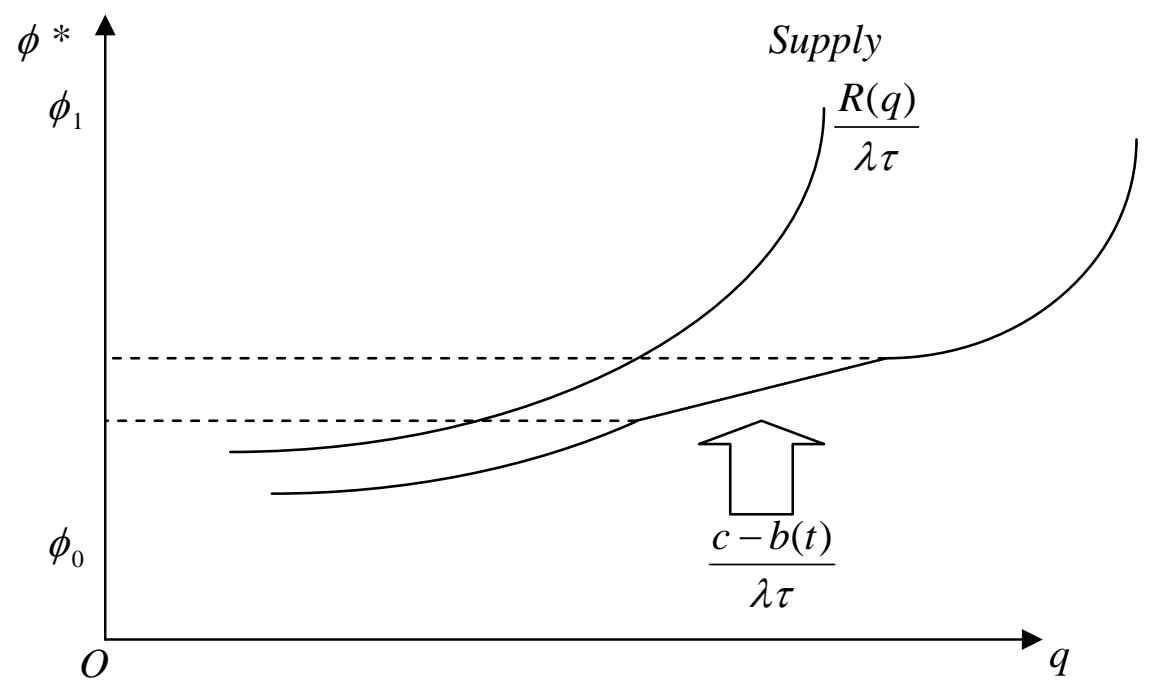


FIGURE 6.B: New Needle Rental Supply Curve with Unlimited Size NEP

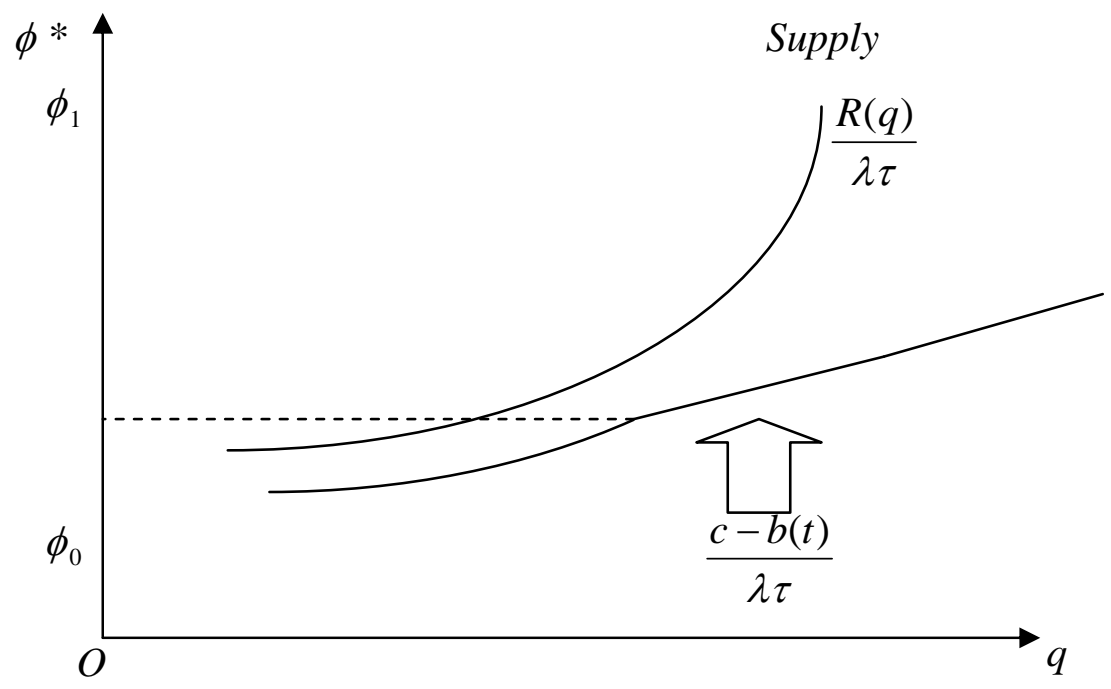

FIGURE 7: Partial Equilibrium Impact of NEP on Equilibrium $\left\{q, \phi^{*}\right\}$

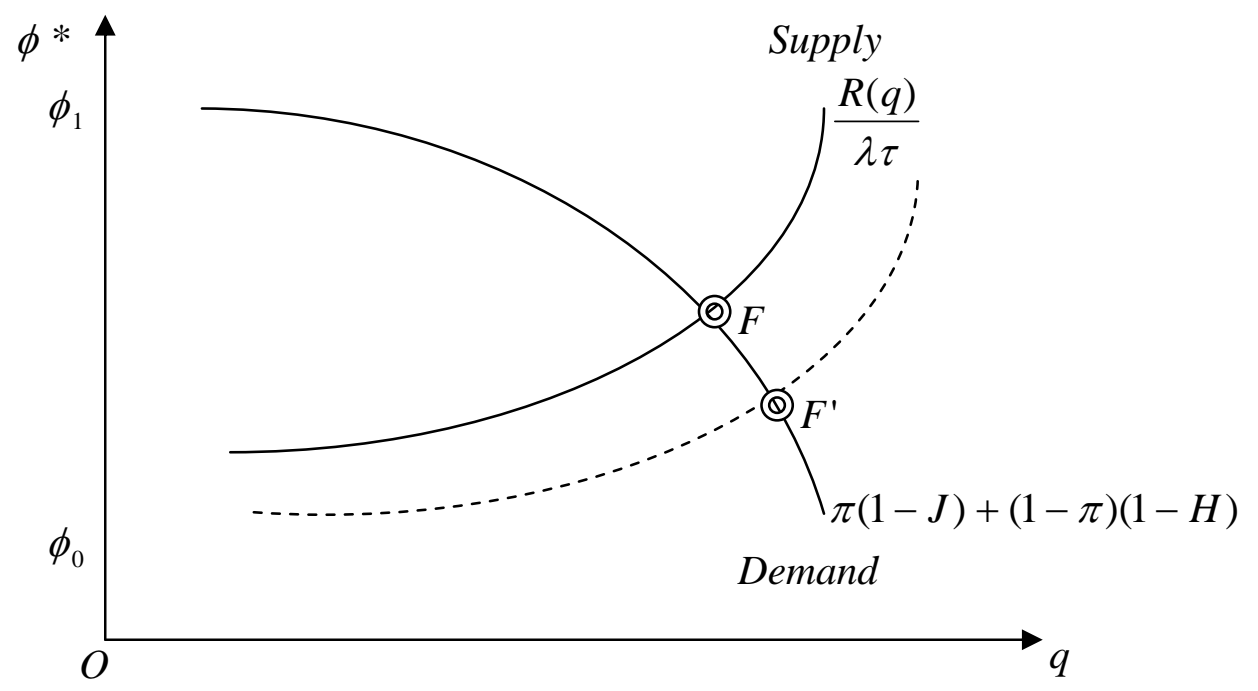


Table 1: Parameter Values for Simulations

\begin{tabular}{|c|c|c|}
\hline Parameter Type & Parameter Symbol & Values Used in Simulations \\
\hline $\begin{array}{l}\text { Mean value of subjective } \\
\text { costs of HIV-infection for } \\
\text { HIV-negative IDU }\end{array}$ & $\frac{1}{\rho}$ & 460,000 \\
\hline $\begin{array}{l}\text { Daily Entry Rate for HIV- } \\
\text { negative IDU }\end{array}$ & $\varepsilon$ & $242 \approx(88400 / 365)$ \\
\hline $\begin{array}{l}\text { Daily Exit Rate for HIV- } \\
\text { negative IDU }\end{array}$ & $\eta$ & $0.000163 \approx(0.0595 / 365)$ \\
\hline $\begin{array}{l}\text { Daily Exit Rate for HIV- } \\
\text { positive IDU }\end{array}$ & $\mu$ & $0.000267 \approx(0.0975 / 365)$ \\
\hline $\begin{array}{l}\text { Proportion of HIV-positive } \\
\text { IDU who know their status }\end{array}$ & $\mathrm{L}$ & $0.05,0.2,0.4,0.6$ \\
\hline $\begin{array}{l}\text { HIV-transmission rate from } \\
\text { single use of infected needle }\end{array}$ & $\tau$ & $1 / 300$ \\
\hline $\begin{array}{c}\text { Elasticity of New Needle } \\
\text { Supply n(q) in Black } \\
\text { Market }\end{array}$ & $\delta$ & $0.0,0.2,0.5,1$ \\
\hline $\begin{array}{l}\text { Scale Parameter for Needle } \\
\text { Supply Function }\end{array}$ & K & 100000 (or calibrated) \\
\hline Bleach Use Rate & $\theta$ & $0.2,0.5,0.8$ \\
\hline $\begin{array}{c}\text { "Size" of NEP } \\
\text { (Proportion of New Needles } \\
\text { Accounted for by NEP) }\end{array}$ & $\mathrm{E}$ & $0.0,0.25,0.5,0.75$ \\
\hline Carrying costs & $\sigma$ & 0 \\
\hline
\end{tabular}

Sources: With the exception of $\mathrm{E}, \delta$, $\mathrm{L}, \sigma$ and $\mathrm{K}$, parameter estimates were derived from existing literature as discussed in greater detail in the Appendix. The value of $\mathrm{K}$ was calibrated so that in the no NEP case, the average number of times a needle was used was roughly the same as reported in the literature for United States. The estimate of parameter $\sigma$ was taken to be zero in our simulations given that carrying costs matter in our model only if the entry/exit rates are endogenous; moreover it helps ensure consistency between the Kaplan-O'Keefe (1993) framework and our model $\mathrm{w}_{\alpha} \ddot{\alpha}$. respect to the simulations. 


\begin{tabular}{|c|c|c|c|c|c|c|c|c|}
\hline \multicolumn{9}{|c|}{ Table 2: Simulation Results: $\theta=0.80 ; L=0.20$} \\
\hline $\begin{array}{c}\text { Needle } \\
\text { Supply } \\
\text { Elasticity }\end{array}$ & $\begin{array}{c}\text { NEP } \\
\text { Size }\end{array}$ & $\alpha$ & $\omega$ & $1+t$ & $\phi^{*}$ & $\lambda$ & I & $\pi$ \\
\hline \multirow[t]{4}{*}{$\delta=0$} & $E=0.00$ & 0.529 & 0.590 & 10.64 & 1030412 & 0.126 & 0.643 & 0.584 \\
\hline & $E=0.25$ & 0.512 & 0.575 & 8.04 & 903527 & 0.122 & 0.628 & 0.567 \\
\hline & $E=0.50$ & 0.474 & 0.542 & 5.46 & 729102 & 0.113 & 0.595 & 0.529 \\
\hline & $E=0.75$ & 0.339 & 0.417 & 2.92 & 455837 & 0.082 & 0.448 & 0.391 \\
\hline \multirow[t]{4}{*}{$\delta=0.2$} & $E=0.00$ & 0.418 & 0.492 & 3.91 & 581216 & 0.100 & 0.539 & 0.473 \\
\hline & $E=0.25$ & 0.367 & 0.445 & 3.19 & 493243 & 0.088 & 0.481 & 0.420 \\
\hline & $E=0.50$ & 0.274 & 0.352 & 2.49 & 388746 & 0.066 & 0.369 & 0.321 \\
\hline & $E=0.75$ & 0.092 & 0.136 & 1.86 & 275094 & 0.022 & 0.129 & 0.112 \\
\hline \multirow[t]{4}{*}{$\delta=0.5$} & $E=0.00$ & 0.161 & 0.225 & 2.04 & 309805 & 0.039 & 0.223 & 0.194 \\
\hline & $E=0.25$ & 0.117 & 0.169 & 1.92 & 286815 & 0.029 & 0.163 & 0.142 \\
\hline & $E=0.50$ & 0.068 & 0.103 & 1.81 & 264835 & 0.017 & 0.096 & 0.083 \\
\hline & $E=0.75$ & 0.021 & 0.034 & 1.72 & 246899 & 0.005 & 0.031 & 0.027 \\
\hline \multirow{4}{*}{$\delta=1$} & $E=0.00$ & 0.039 & 0.061 & 1.75 & 253374 & 0.010 & 0.055 & 0.048 \\
\hline & $E=0.25$ & 0.030 & 0.047 & 1.74 & 250037 & 0.007 & 0.043 & 0.037 \\
\hline & $E=0.50$ & 0.021 & 0.034 & 1.67 & 235498 & 0.005 & 0.030 & 0.026 \\
\hline & $E=0.75$ & 0.013 & 0.020 & 1.51 & 190924 & 0.003 & 0.016 & 0.014 \\
\hline
\end{tabular}




\begin{tabular}{|c|c|c|c|c|c|c|c|c|}
\hline \multicolumn{9}{|c|}{ Table 3: Simulation Results: $\theta=0.50 ; L=0.20$} \\
\hline $\begin{array}{l}\text { Needle } \\
\text { Supply } \\
\text { Elasticity }\end{array}$ & $\begin{array}{c}\text { NEP } \\
\text { Size } \\
\end{array}$ & $\alpha$ & $\omega$ & $1+t$ & $\phi^{*}$ & $\lambda$ & I & $\pi$ \\
\hline \multirow{4}{*}{$\delta=0$} & $E=0.00$ & 0.796 & 0.834 & 9.55 & 954288 & 0.448 & 0.909 & 0.830 \\
\hline & $E=0.25$ & 0.786 & 0.827 & 7.19 & 824561 & 0.442 & 0.905 & 0.821 \\
\hline & $E=0.50$ & 0.764 & 0.811 & 4.83 & 644075 & 0.430 & 0.892 & 0.802 \\
\hline & $E=0.75$ & 0.667 & 0.746 & 2.51 & 351867 & 0.375 & 0.804 & 0.715 \\
\hline \multirow[t]{4}{*}{$\delta=0.2$} & $E=0.00$ & 0.696 & 0.765 & 2.83 & 404818 & 0.391 & 0.833 & 0.741 \\
\hline & $E=0.25$ & 0.643 & 0.730 & 2.31 & 317053 & 0.362 & 0.779 & 0.692 \\
\hline & $E=0.50$ & 0.530 & 0.652 & 2.81 & 216625 & 0.300 & 0.657 & 0.585 \\
\hline & $E=0.75$ & 0.245 & 0.405 & 1.38 & 119078 & 0.143 & 0.322 & 0.289 \\
\hline \multirow{4}{*}{$\delta=0.5$} & $E=0.00$ & 0.290 & 0.453 & 1.42 & 128351 & 0.168 & 0.377 & 0.338 \\
\hline & $E=0.25$ & 0.218 & 0.374 & 1.35 & 114044 & 0.127 & 0.288 & 0.259 \\
\hline & $E=0.50$ & 0.134 & 0.260 & 1.29 & 100635 & 0.079 & 0.180 & 0.162 \\
\hline & $E=0.75$ & 0.046 & 0.105 & 1.23 & 89472 & 0.028 & 0.063 & 0.057 \\
\hline \multirow{4}{*}{$\delta=1$} & $E=0.00$ & 0.062 & 0.137 & 1.24 & 91324 & 0.037 & 0.085 & 0.077 \\
\hline & $E=0.25$ & 0.048 & 0.109 & 1.23 & 89695 & 0.029 & 0.066 & 0.060 \\
\hline & $E=0.50$ & 0.034 & 0.078 & 1.22 & 88011 & 0.020 & 0.046 & 0.041 \\
\hline & $E=0.75$ & 0.017 & 0.042 & 1.21 & 86265 & 0.010 & 0.024 & 0.022 \\
\hline
\end{tabular}




\begin{tabular}{|c|c|c|c|c|c|c|c|c|}
\hline \multicolumn{9}{|c|}{ Table 4: Simulation Results $\theta=0.20 ; L=0.20$} \\
\hline $\begin{array}{c}\text { Supply } \\
\text { Elasticity }\end{array}$ & $\begin{array}{l}\text { NEP } \\
\text { Size }\end{array}$ & $\alpha$ & $\omega$ & $1+t$ & $\phi^{*}$ & $\lambda$ & $I$ & $\pi$ \\
\hline \multirow[t]{4}{*}{$\delta=0$} & $E=0.00$ & 0.870 & 0.896 & 9.30 & 935328 & 0.771 & 0.977 & 0.893 \\
\hline & $E=0.25$ & 0.863 & 0.892 & 6.99 & 804705 & 0.765 & 0.976 & 0.887 \\
\hline & $E=0.50$ & 0.847 & 0.881 & 4.69 & 622203 & 0.750 & 0.969 & 0.874 \\
\hline & $E=0.75$ & 0.775 & 0.837 & 2.41 & 322303 & 0.683 & 0.907 & 0.811 \\
\hline \multirow[t]{4}{*}{$\delta=0.2$} & $E=0.00$ & 0.784 & 0.843 & 2.52 & 343425 & 0.692 & 0.917 & 0.820 \\
\hline & $E=0.25$ & 0.735 & 0.815 & 2.07 & 256898 & 0.649 & 0.867 & 0.776 \\
\hline & $E=0.50$ & 0.619 & 0.750 & 1.64 & 161124 & 0.551 & 0.746 & 0.670 \\
\hline & $E=0.75$ & 0.299 & 0.526 & 1.27 & 77834 & 0.274 & 0.385 & 0.348 \\
\hline \multirow[t]{4}{*}{$\delta=0.5$} & $E=0.00$ & 0.330 & 0.555 & 1.29 & 82023 & 0.302 & 0.421 & 0.381 \\
\hline & $E=0.25$ & 0.250 & 0.476 & 1.24 & 71859 & 0.230 & 0.324 & 0.294 \\
\hline & $E=0.50$ & 0.155 & 0.352 & 1.19 & 62522 & 0.144 & 0.205 & 0.186 \\
\hline & $E=0.75$ & 0.055 & 0.157 & 1.14 & 54822 & 0.051 & 0.074 & 0.067 \\
\hline \multirow[t]{4}{*}{$\delta=1$} & $E=0.00$ & 0.069 & 0.191 & 1.15 & 55815 & 0.065 & 0.093 & 0.085 \\
\hline & $E=0.25$ & 0.054 & 0.154 & 1.14 & 54751 & 0.050 & 0.072 & 0.066 \\
\hline & $E=0.50$ & 0.037 & 0.112 & 1.13 & 53652 & 0.035 & 0.050 & 0.046 \\
\hline & $E=0.75$ & 0.019 & 0.061 & 1.13 & 52511 & 0.018 & 0.026 & 0.024 \\
\hline
\end{tabular}




\begin{tabular}{|c|c|c|c|c|c|c|c|c|}
\hline \multicolumn{9}{|c|}{ Table 5: Simulation Results $\theta=0.50 ; L=0.05$} \\
\hline $\begin{array}{c}\text { Supply } \\
\text { Elasticity }\end{array}$ & $\begin{array}{l}N E P \\
\text { Size }\end{array}$ & $\alpha$ & $\omega$ & $1+t$ & $\phi^{*}$ & $\lambda$ & $I$ & $\pi$ \\
\hline \multirow[t]{4}{*}{$\delta=0$} & $E=0.00$ & 0.826 & 0.834 & 9.533 & 1017617 & 0.449 & 0.910 & 0.833 \\
\hline & $E=0.25$ & 0.819 & 0.827 & 7.171 & 886842 & 0.445 & 0.905 & 0.826 \\
\hline & $E=0.50$ & 0.802 & 0.812 & 4.814 & 703882 & 0.435 & 0.893 & 0.810 \\
\hline & $E=0.75$ & 0.730 & 0.747 & 2.480 & 400355 & 0.392 & 0.821 & 0.740 \\
\hline \multirow[t]{4}{*}{$\delta=0.2$} & $E=0.00$ & 0.748 & 0.763 & 2.740 & 445944 & 0.403 & 0.840 & 0.757 \\
\hline & $E=0.25$ & 0.705 & 0.725 & 2.222 & 350661 & 0.378 & 0.793 & 0.716 \\
\hline & $E=0.50$ & 0.608 & 0.637 & 1.726 & 236537 & 0.325 & 0.684 & 0.620 \\
\hline & $E=0.75$ & 0.308 & 0.354 & 1.316 & 118846 & 0.164 & 0.349 & 0.319 \\
\hline \multirow[t]{4}{*}{$\delta=0.5$} & $E=0.00$ & 0.346 & 0.392 & 1.342 & 127054 & 0.185 & 0.392 & 0.358 \\
\hline & $E=0.25$ & 0.264 & 0.309 & 1.290 & 110822 & 0.141 & 0.300 & 0.274 \\
\hline & $E=0.50$ & 0.164 & 0.201 & 1.242 & 95874 & 0.088 & 0.187 & 0.171 \\
\hline & $E=0.75$ & 0.057 & 0.074 & 1.203 & 83760 & 0.031 & 0.065 & 0.060 \\
\hline \multirow[t]{4}{*}{$\delta=1$} & $E=0.00$ & 0.075 & 0.096 & 1.209 & 85596 & 0.040 & 0.086 & 0.079 \\
\hline & $E=0.25$ & 0.058 & 0.075 & 1.204 & 83895 & 0.031 & 0.067 & 0.061 \\
\hline & $E=0.50$ & 0.040 & 0.053 & 1.198 & 82144 & 0.022 & 0.046 & 0.042 \\
\hline & $E=0.75$ & 0.021 & 0.028 & 1.192 & 80336 & 0.011 & 0.024 & 0.022 \\
\hline
\end{tabular}




\begin{tabular}{|c|c|c|c|c|c|c|c|c|}
\hline \multicolumn{9}{|c|}{ Table 6: Simulation Results $\theta=0.50 ; L=0.20$} \\
\hline $\begin{array}{c}\text { Supply } \\
\text { Elasticity }\end{array}$ & $\begin{array}{c}N E P \\
\text { Size }\end{array}$ & $\alpha$ & $\omega$ & $1+t$ & $\phi^{*}$ & $\lambda$ & $I$ & $\pi$ \\
\hline \multirow[t]{4}{*}{$\delta=0$} & $E=0.00$ & 0.796 & 0.834 & 9.546 & 954288 & 0.448 & 0.910 & 0.830 \\
\hline & $E=0.25$ & 0.786 & 0.827 & 7.185 & 824561 & 0.442 & 0.905 & 0.822 \\
\hline & $E=0.50$ & 0.764 & 0.812 & 4.830 & 644075 & 0.430 & 0.892 & 0.802 \\
\hline & $E=0.75$ & 0.667 & 0.746 & 2.507 & 351867 & 0.375 & 0.804 & 0.715 \\
\hline \multirow[t]{4}{*}{$\delta=0.2$} & $E=0.00$ & 0.696 & 0.765 & 2.830 & 404818 & 0.391 & 0.833 & 0.741 \\
\hline & $E=0.25$ & 0.643 & 0.730 & 2.312 & 317053 & 0.362 & 0.779 & 0.692 \\
\hline & $E=0.50$ & 0.530 & 0.652 & 1.816 & 216625 & 0.300 & 0.657 & 0.585 \\
\hline & $E=0.75$ & 0.245 & 0.405 & 1.375 & 119078 & 0.148 & 0.322 & 0.289 \\
\hline \multirow[t]{4}{*}{$\delta=0.5$} & $E=0.00$ & 0.290 & 0.453 & 1.418 & 128351 & 0.168 & 0.377 & 0.338 \\
\hline & $E=0.25$ & 0.218 & 0.374 & 1.351 & 114044 & 0.127 & 0.288 & 0.259 \\
\hline & $E=0.50$ & 0.134 & 0.260 & 1.286 & 100635 & 0.079 & 0.180 & 0.162 \\
\hline & $E=0.75$ & 0.046 & 0.105 & 1.229 & 89472 & 0.028 & 0.063 & 0.057 \\
\hline \multirow{4}{*}{$\delta=1$} & $E=0.00$ & 0.062 & 0.137 & 1.239 & 91324 & 0.037 & 0.085 & 0.077 \\
\hline & $E=0.25$ & 0.048 & 0.109 & 1.230 & 89695 & 0.029 & 0.066 & 0.060 \\
\hline & $E=0.50$ & 0.034 & 0.078 & 1.221 & 88011 & 0.020 & 0.046 & 0.041 \\
\hline & $E=0.75$ & 0.017 & 0.042 & 1.212 & 86265 & 0.010 & 0.024 & 0.022 \\
\hline
\end{tabular}




\begin{tabular}{|c|c|c|c|c|c|c|c|c|}
\hline \multicolumn{9}{|c|}{ Table 7: Simulation Results $\theta=0.50 ; L=0.40$} \\
\hline $\begin{array}{c}\text { Needle } \\
\text { Supply } \\
\text { Elasticity }\end{array}$ & $\begin{array}{c}N E P \\
\text { Size }\end{array}$ & $\alpha$ & $\omega$ & $1+t$ & $\phi^{*}$ & $\lambda$ & $I$ & $\pi$ \\
\hline \multirow[t]{4}{*}{$\delta=0$} & $E=0.00$ & 0.737 & 0.834 & 9.57 & 855094 & 0.444 & 0.909 & 0.824 \\
\hline & $E=0.25$ & 0.722 & 0.827 & 7.21 & 728095 & 0.437 & 0.905 & 0.812 \\
\hline & $E=0.50$ & 0.687 & 0.811 & 4.86 & 554092 & 0.419 & 0.889 & 0.785 \\
\hline & $E=0.75$ & 0.545 & 0.744 & 2.56 & 289925 & 0.343 & 0.774 & 0.667 \\
\hline \multirow[t]{4}{*}{$\delta=0.2$} & $E=0.00$ & 0.596 & 0.769 & 2.99 & 349249 & 0.370 & 0.822 & 0.711 \\
\hline & $E=0.25$ & 0.530 & 0.736 & 2.46 & 275182 & 0.334 & 0.759 & 0.652 \\
\hline & $E=0.50$ & 0.407 & 0.667 & 1.95 & 195753 & 0.264 & 0.621 & 0.507 \\
\hline & $E=0.75$ & 0.170 & 0.441 & 1.45 & 121883 & 0.117 & 0.292 & 0.254 \\
\hline \multirow[t]{4}{*}{$\delta=0.5$} & $E=0.00$ & 0.214 & 0.500 & 1.52 & 131642 & 0.146 & 0.361 & 0.312 \\
\hline & $E=0.25$ & 0.159 & 0.425 & 1.43 & 119677 & 0.110 & 0.275 & 0.239 \\
\hline & $E=0.50$ & 0.096 & 0.308 & 1.35 & 108199 & 0.068 & 0.172 & 0.151 \\
\hline & $E=0.75$ & 0.033 & 0.132 & 1.27 & 98335 & 0.024 & 0.061 & 0.054 \\
\hline \multirow[t]{4}{*}{$\delta=1$} & $E=0.00$ & 0.046 & 0.174 & 1.28 & 100172 & 0.033 & 0.084 & 0.074 \\
\hline & $E=0.25$ & 0.035 & 0.140 & 1.27 & 98662 & 0.025 & 0.065 & 0.058 \\
\hline & $E=0.50$ & 0.024 & 0.101 & 1.26 & 97094 & 0.018 & 0.045 & 0.040 \\
\hline & $E=0.75$ & 0.013 & 0.055 & 1.24 & 95460 & 0.009 & 0.024 & 0.021 \\
\hline
\end{tabular}




\begin{tabular}{|c|c|c|c|c|c|c|c|c|}
\hline \multicolumn{9}{|c|}{ Table 8: Simulation Results $\theta=0.50 ; L=0.60$} \\
\hline $\begin{array}{c}\text { Needle } \\
\text { Supply } \\
\text { Elasticity }\end{array}$ & $\begin{array}{c}\text { NEP } \\
\text { Size }\end{array}$ & $\alpha$ & $\omega$ & $1+t$ & $\phi^{*}$ & $\lambda$ & I & $\pi$ \\
\hline \multirow[t]{4}{*}{$\delta=0$} & $E=0.00$ & 0.635 & 0.834 & 9.614 & 733248 & 0.437 & 0.909 & 0.813 \\
\hline & $E=0.25$ & 0.611 & 0.827 & 7.260 & 612742 & 0.427 & 0.904 & 0.797 \\
\hline & $E=0.50$ & 0.557 & 0.810 & 4.920 & 453623 & 0.401 & 0.885 & 0.758 \\
\hline & $E=0.75$ & 0.378 & 0.740 & 2.637 & 239557 & 0.299 & 0.737 & 0.603 \\
\hline \multirow[t]{4}{*}{$\delta=0.2$} & $E=0.00$ & 0.448 & 0.772 & 3.190 & 296989 & 0.342 & 0.810 & 0.670 \\
\hline & $E=0.25$ & 0.378 & 0.741 & 2.642 & 240148 & 0.300 & 0.738 & 0.603 \\
\hline & $E=0.50$ & 0.270 & 0.674 & 2.087 & 181873 & 0.227 & 0.590 & 0.481 \\
\hline & $E=0.75$ & 0.103 & 0.454 & 1.523 & 127313 & 0.096 & 0.268 & 0.224 \\
\hline \multirow[t]{4}{*}{$\delta=0.5$} & $E=0.00$ & 0.139 & 0.525 & 1.627 & 136746 & 0.126 & 0.348 & 0.288 \\
\hline & $E=0.25$ & 0.102 & 0.450 & 1.519 & 126959 & 0.094 & 0.265 & 0.221 \\
\hline & $E=0.50$ & 0.061 & 0.333 & 1.409 & 117371 & 0.058 & 0.166 & 0.140 \\
\hline & $E=0.75$ & 0.021 & 0.147 & 1.307 & 108911 & 0.020 & 0.059 & 0.050 \\
\hline \multirow[t]{4}{*}{$\delta=1$} & $E=0.00$ & 0.030 & 0.196 & 1.328 & 110672 & 0.029 & 0.083 & 0.071 \\
\hline & $E=0.25$ & 0.023 & 0.159 & 1.312 & 109318 & 0.022 & 0.064 & 0.055 \\
\hline & $E=0.50$ & 0.016 & 0.116 & 1.294 & 107908 & 0.015 & 0.045 & 0.038 \\
\hline & $E=0.75$ & 0.008 & 0.064 & 1.276 & 106432 & 0.008 & 0.023 & 0.020 \\
\hline
\end{tabular}




\begin{tabular}{|c|c|c|c|c|c|c|}
\hline $\begin{array}{l}\text { Needle } \\
\text { Supply } \\
\text { Elasticity }\end{array}$ & $\begin{array}{c}\text { NEP } \\
\text { Size }\end{array}$ & $\begin{array}{l}\text { Kaplan- } \\
\text { O'Keefe } \\
\quad \bar{n}\end{array}$ & $\begin{array}{c}\text { Kaplan- } \\
\text { O’Keefe } \\
\quad \bar{p}\end{array}$ & $\lambda$ & $I$ & $\pi$ \\
\hline \multirow[t]{4}{*}{$\delta=0$} & $E=0.00$ & 0.678 & 0.629 & 0.126 & 0.643 & 0.584 \\
\hline & $E=0.25$ & 0.647 & 0.618 & 0.122 & 0.628 & 0.567 \\
\hline & $E=0.50$ & 0.589 & 0.595 & 0.113 & 0.595 & 0.529 \\
\hline & $E=0.75$ & 0.459 & 0.534 & 0.082 & 0.448 & 0.391 \\
\hline \multirow[t]{4}{*}{$\delta=0.2$} & $E=0.00$ & 0.678 & 0.629 & 0.100 & 0.539 & 0.473 \\
\hline & $E=0.25$ & 0.601 & 0.600 & 0.088 & 0.481 & 0.420 \\
\hline & $E=0.50$ & 0.499 & 0.555 & 0.066 & 0.369 & 0.321 \\
\hline & $E=0.75$ & 0.369 & 0.480 & 0.022 & 0.129 & 0.112 \\
\hline \multirow[t]{4}{*}{$\delta=0.5$} & $E=0.00$ & 0.678 & 0.629 & 0.039 & 0.223 & 0.194 \\
\hline & $E=0.25$ & 0.555 & 0.581 & 0.029 & 0.163 & 0.142 \\
\hline & $E=0.50$ & 0.446 & 0.527 & 0.017 & 0.096 & 0.083 \\
\hline & $E=0.75$ & 0.352 & 0.468 & 0.005 & 0.031 & 0.027 \\
\hline \multirow[t]{4}{*}{$\delta=1$} & $E=0.00$ & 0.678 & 0.629 & 0.010 & 0.055 & 0.048 \\
\hline & $E=0.25$ & 0.544 & 0.576 & 0.007 & 0.043 & 0.037 \\
\hline & $E=0.50$ & 0.430 & 0.518 & 0.005 & 0.030 & 0.026 \\
\hline & $E=0.75$ & 0.321 & 0.445 & 0.003 & 0.016 & 0.014 \\
\hline
\end{tabular}

Note: The version of the Kaplan-O'Keefe model used for the simulations is more fully described in Technical Appendix A, in equations (A1) and (A2) and the steady state conditions (A4) and (A6). 
Table 10: Steady state HIV prevalence among IDU corresponding to before-NEP and afterNEP terminal needle infection rates observed in New Haven: A comparison of the Behavioral and the Kaplan-O'Keefe Models

\begin{tabular}{|c|c|c|c|c|}
\hline \multirow{2}{*}{$\begin{array}{l}\text { Share of HIV+ } \\
\text { who know their } \\
\text { status (L) and } \\
\text { Bleach Rate }(\theta)\end{array}$} & \multicolumn{2}{|c|}{$\begin{array}{l}\text { Steady State HIV Prevalence in } \\
\text { IDU } \\
\text { Predictions from Behavioral } \\
\text { Model (\%) }\end{array}$} & \multicolumn{2}{|c|}{$\begin{array}{c}\text { Steady State HIV Prevalence in } \\
\text { IDU } \\
\text { Predictions from Kaplan- } \\
\text { O’Keefe (\%) }\end{array}$} \\
\hline & Before NEP & After NEP & Before NEP & After NEP \\
\hline$L=0.05 ; \theta=0.80$ & 61.88 & 35.88 & 62.92 & 48.15-48.89 \\
\hline$L=0.20 ; \theta=0.80$ & 61.79 & 34.83 & 62.92 & 48.96-49.69 \\
\hline$L=0.40 ; \theta=0.80$ & 61.57 & 33.26 & 62.92 & 49.93-50.66 \\
\hline$L=0.60 ; \theta=0.80$ & 61.31 & 31.61 & 62.92 & 50.78-51.91 \\
\hline$L=0.05 ; \theta=0.50$ & 60.77 & 36.50 & 85.17 & 76.77-81.95 \\
\hline$L=0.20 ; \theta=0.50$ & 59.64 & 35.85 & 85.17 & 77.31-81.99 \\
\hline$L=0.40 ; \theta=0.50$ & 57.51 & 34.57 & 85.17 & 77.99-82.06 \\
\hline$L=0.60 ; \theta=0.50$ & 54.61 & 32.94 & 85.17 & 78.64-82.16 \\
\hline
\end{tabular}

Note: Before- and after-NEP estimates for the Kaplan-O'Keefe framework are derived from equation (A6) in Technical Appendix A, namely $\bar{p}=\frac{\alpha(1-\theta)-\mu \theta-\mu \kappa}{(\alpha+\mu)(1-\theta)}$. The value of the parameter $\mu$ was taken to be the estimated daily exit rate from the population of 0.000267 . The value of parameter $\kappa$ (NEP size in the Kaplan-O'Keefe framework) was taken to be zero initially. Its (equilibrium) size was taken to be $\frac{E}{(1+t)}$ as derived in the text, where $\mathrm{E}$ is the NEP size required to achieve an observed terminal needle infection rate of 0.40 , similar to the proportion observed by 
Kaplan and O’Keefe in their New Haven study. Because $\alpha=\frac{\gamma \tau}{\varepsilon}$, and each IDU is assumed to inject once daily in our model, we have that $\frac{\gamma}{\varepsilon}=1$, and hence $\alpha=\tau=1 / 300$.

Before-NEP and after NEP prevalence of HIV among IDU in the behavioral model are derived as follows. First, assuming no NEP, the supply function scale parameter was calibrated so as to yield a steady state outcome of a terminal needle infection rate $I(t)=0.670$. This also yielded an associated steady state HIV prevalence rate among IDU (pre-NEP). Next, holding K to be the same, we estimated the size of the needle exchange program (in term of the proportion of new needles accounted for the NEP) required for achieving the terminal needle infection rate of 0.40 . The steady state HIV prevalence rate among IDU (post-NEP) corresponded to this new equilibrium.

Obviously, the size of the NEP required to achieve equilibrium at these terminal needle infection rates was directly related to the elasticity of new needle supplies in the black market. The range of estimates of IDU prevalence in column (4) of the above table reflects estimates corresponding to equilibrium NEP sizes for alternative elasticities of supply of new needles. 


\section{TECHNICAL APPENDIX A}

$\underline{\text { Result 1: }} \alpha \leq \omega$

Proof:

From equation (4) in the paper, we have that

$q\left(\phi^{*}\right)=\pi\left(1-J\left(\phi^{*}\right)\right)+(1-\pi)\left(1-H\left(\phi^{*}\right)\right)$

Then, by the assumption of stochastic dominance $J(\phi) \geq H(\phi)$ we have

$$
q\left(\phi^{*}\right)=\pi\left(1-J\left(\phi^{*}\right)\right)+(1-\pi)\left(1-H\left(\phi^{*}\right)\right) \geq 1-J\left(\phi^{*}\right)
$$

Thus

(A2) $\frac{1-J\left(\phi^{*}\right)}{q\left(\phi^{*}\right)} \leq 1$; and $\frac{J\left(\phi^{*}\right)}{1-q\left(\phi^{*}\right)} \geq 1$

Using the inequalities in (A2) we have

(A3) $\frac{J\left(\phi^{*}\right)}{1-q\left(\phi^{*}\right)} \geq \frac{1-J\left(\phi^{*}\right)}{q\left(\phi^{*}\right)}$

Multiplying both sides of (A3) by $\pi$ and noting that $\alpha=\frac{\pi\left(1-J\left(\phi^{*}\right)\right)}{q\left(\phi^{*}\right)}$ and $\omega=\frac{\pi J\left(\phi^{*}\right)}{1-q\left(\phi^{*}\right)}$ yields the desired result.

Result 2: $\alpha$ and $\omega$ are non-increasing in $\phi^{*}$.

We note that $\alpha=\frac{\pi\left(1-J\left(\phi^{*}\right)\right)}{q\left(\phi^{*}\right)}$ and $\omega=\frac{\pi J\left(\phi^{*}\right)}{1-q\left(\phi^{*}\right)}$

Proof: Taking derivatives of $\alpha$ and $\omega$ with respect to $\phi^{*}$, respectively, we have

(A4) $\frac{\partial \omega}{\partial \phi}=\frac{\pi(1-\pi)}{\left(1-q\left(\phi^{*}\right)\right)^{2}} J\left(\phi^{*}\right) j\left(\phi^{*}\right)\left(\frac{H\left(\phi^{*}\right)}{J\left(\phi^{*}\right)}-\frac{h\left(\phi^{*}\right)}{j\left(\phi^{*}\right)}\right)$, and 


$$
\frac{\partial \alpha}{\partial \phi}=\frac{\pi(1-\pi)}{q\left(\phi^{*}\right)^{2}}\left(1-J\left(\phi^{*}\right)\right) j\left(\phi^{*}\right)\left(\frac{h\left(\phi^{*}\right)}{j\left(\phi^{*}\right)}-\frac{1-H\left(\phi^{*}\right)}{1-J\left(\phi^{*}\right)}\right)
$$

Observe that $\frac{H\left(\phi^{*}\right)}{J\left(\phi^{*}\right)}$ is a weighted average of $\frac{h(\phi)}{j(\phi)}$ for $\phi<\phi^{*}$. By Monotone Likelihood Ratio Property (MLRP), we have that $\frac{h(\phi)}{j(\phi)} \leq \frac{h\left(\phi^{*}\right)}{j\left(\phi^{*}\right)}$ for $\phi<\phi^{*}$. Combining these two results, we have

$$
\frac{H\left(\phi^{*}\right)}{J\left(\phi^{*}\right)} \leq \frac{h\left(\phi^{*}\right)}{j\left(\phi^{*}\right)}
$$

It immediately follows from (A4) that $\frac{\partial \omega}{\partial \phi^{*}} \leq 0$.

Note that $\frac{h\left(\phi^{*}\right)}{j\left(\phi^{*}\right)} \leq \frac{h(\phi)}{j(\phi)}$ for $\phi>\phi^{*}$, and similarly $\frac{1-H\left(\phi^{*}\right)}{1-J\left(\phi^{*}\right)} \geq \frac{h\left(\phi^{*}\right)}{j\left(\phi^{*}\right)}$. Thus, it follows that $\frac{\partial \alpha}{\partial \phi^{*}} \leq 0$ QED.

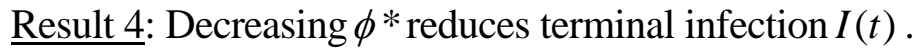

Proof: Note that $I(t)=1-(1-\alpha) e^{-t \omega}$. Taking natural logs on both sides we have

$$
Y \equiv \ln (1-I(t))=\ln \left(1-\alpha\left(\phi^{*}\right)\right)-t\left(\phi^{*}\right) \omega\left(\phi^{*}\right)
$$

Decreasing $\phi^{*}$ will decrease the terminal infection rate if and only if it increases $\mathrm{Y}$, so we can concentrate on $\mathrm{Y}$.

$$
\text { Now } \alpha=\frac{\pi\left(1-J\left(\phi^{*}\right)\right)}{q\left(\phi^{*}\right)} \text { and } q\left(\phi^{*}\right)=\pi\left(1-J\left(\phi^{*}\right)\right)+(1-\pi)\left(1-H\left(\phi^{*}\right)\right) \text { [Equations (4) and (6) }
$$

in the paper]. Combining the two equations yields

$$
(1-\alpha)=\frac{(1-\pi)\left(1-H\left(\phi^{*}\right)\right.}{q\left(\phi^{*}\right)}
$$

Differentiating $\ln \left(1-\alpha\left(\phi^{*}\right)\right.$ with respect to $\phi^{*}$, we get 


$$
\frac{\partial}{\partial \phi^{*}} \ln (1-\alpha)=\frac{\pi j\left(\phi^{*}\right)}{q\left(\phi^{*}\right)}-\frac{\pi h\left(\phi^{*}\right)\left(1-J\left(\phi^{*}\right)\right)}{q\left(\phi^{*}\right)\left(1-H\left(\phi^{*}\right)\right)}
$$

Where, in addition to (A7), we have used the results from (A4).

Recall that, $t \omega=\frac{1-q\left(\phi^{*}\right)}{q\left(\phi^{*}\right)} \frac{\pi J\left(\phi^{*}\right)}{1-q\left(\phi^{*}\right)}=\frac{\pi J\left(\phi^{*}\right)}{q\left(\phi^{*}\right)}$ [equation (9) in the paper]. Then noting $q\left(\phi^{*}\right)=\pi\left(1-J\left(\phi^{*}\right)\right)+(1-\pi)\left(1-H\left(\phi^{*}\right)\right)$ and differentiating $t\left(\phi^{*}\right) \omega\left(\phi^{*}\right)$ with respect to $\phi^{*}$, we get

$$
\frac{\partial}{\partial \phi^{*}} t \omega=\frac{\pi j\left(\phi^{*}\right)}{q\left(\phi^{*}\right)}+\frac{\pi J\left(\phi^{*}\right)}{q\left(\phi^{*}\right)^{2}}\left(\pi j\left(\phi^{*}\right)+(1-\pi) h\left(\phi^{*}\right)\right)
$$

The first term in expressions (A8) and (A9) is the same. However, the second term is negative for $\frac{\partial}{\partial \phi^{*}} \ln (1-\alpha)$ and positive for $\frac{\partial}{\partial \phi^{*}} t \omega$. Thus $\frac{\partial Y}{\partial \phi^{*}}<0$, and we have that decreasing $\phi^{*}$ results in a decrease the terminal infection rate. QED

Technical Note: To begin our analysis of the impact of $\phi^{*}$ on the average infection rate we examine the properties of the function $g(x)$ where

$$
g(x)=\frac{1-e^{-x}}{x}
$$

Note in particular that:

$$
\begin{aligned}
& 0<g(x)<1 \quad \text { For all } x \\
& g^{\prime}(x)<0 \quad \text { For all } x \\
& \operatorname{Lim}_{x \rightarrow 0} g(x)=1 ; \operatorname{Lim}_{x \rightarrow 0} g^{\prime}(x)=-\frac{1}{2} ; \operatorname{Lim}_{x \rightarrow \infty} g(x)=\operatorname{Lim}_{x \rightarrow \infty} g^{\prime}(x)=0
\end{aligned}
$$


The average needle infection rate $\lambda(t)$ is given by $\lambda(t)=1-(1-\alpha) \frac{1-e^{-t \omega}}{t \omega}$. We can write

$$
y \equiv \ln (1-\lambda(t))=\ln (1-\alpha)+\ln g(t \omega)
$$

From (A12) we have that in $(\ln g(t \omega), \ln (1-\alpha))$ space, the combinations that yield equal values of $\mathbf{y}$ are straight lines with a slope of minus one, as in Figure A1. Note also that movements in the northeast direction indicate lower levels of average needle infection. ${ }^{12}$

Feasible combinations of $\ln g(t \omega)$ and $\ln (1-\alpha)$ must satisfy the requirement that the level of $\phi^{*}$ to which they correspond is identical. Thus, we graph $(\ln (g(t \omega)), \ln (1-\alpha))$ parametrically as a function of $\phi^{*}$. At $\phi^{*}=\phi_{0}, t \omega=0$ and so $g(t \omega)=1$ and $\ln (g(t \omega))=0$. As $\phi^{*}$ increases, $t \omega$ increases and, therefore, $\ln (g(t \omega))$ decreases, from (A11). Since $t \omega$ tends to infinity as $\phi^{*} \rightarrow \phi_{1}$, $g(t \omega)$ approaches zero, by (A11). Thus, as $\phi^{*} \rightarrow \phi_{1}, \ln (g(t \omega))$ tends to negative infinity.

Now consider what happens to $\ln (1-\alpha)$ as $\phi^{*}$ changes. Since $\ln (1-\alpha)$ equals $\ln \frac{(1-\pi)(1-H)}{q}$ and moreover, $H\left(\phi_{0}\right)=1-q\left(\phi_{0}\right)=0$, we have that when $\phi^{*}$ equals $\phi_{0}, \ln (1-\alpha)$ equals $\ln (1-\pi)$, which is less than zero. As $\phi^{*}$ rises from $\phi_{0}$, we know, from (A12) that $\ln (1-\alpha)$ will also increase. It is, however, bounded from above by zero.

\footnotetext{
${ }^{12}$ Note that $Y \equiv \ln (1-I(t))=\ln \left(1-\alpha\left(\phi^{*}\right)\right)-t\left(\phi^{*}\right) \omega\left(\phi^{*}\right)$. The assertion then follows from the observation that reductions in $\phi^{*}$ reduce $\alpha$ and $t \omega$, thereby raising both $(1-\alpha)$ and $g(t \omega)$.
} 


\section{FIGURE A1: Iso-average Infection Curves}

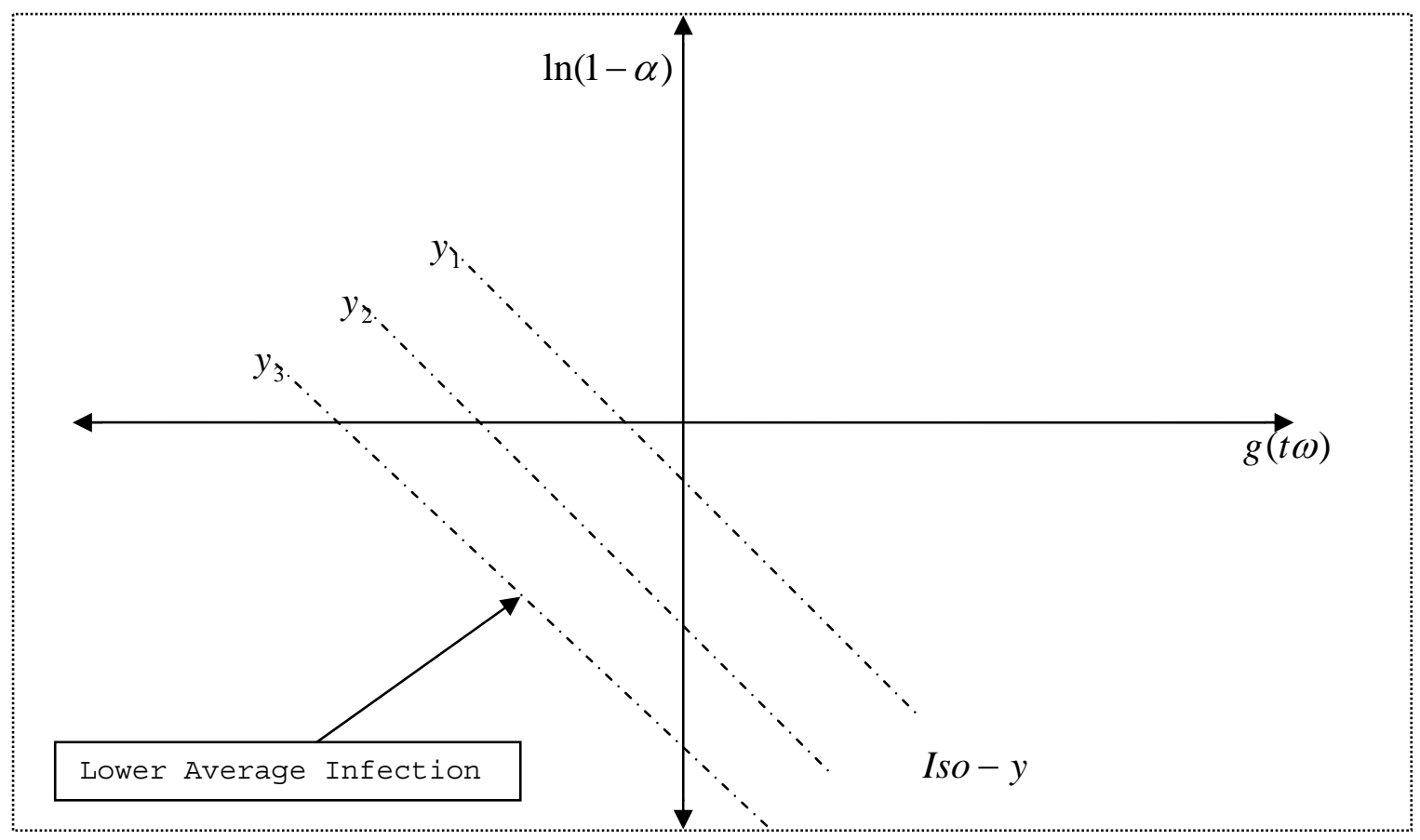

FIGURE A2: The Constraint Curve

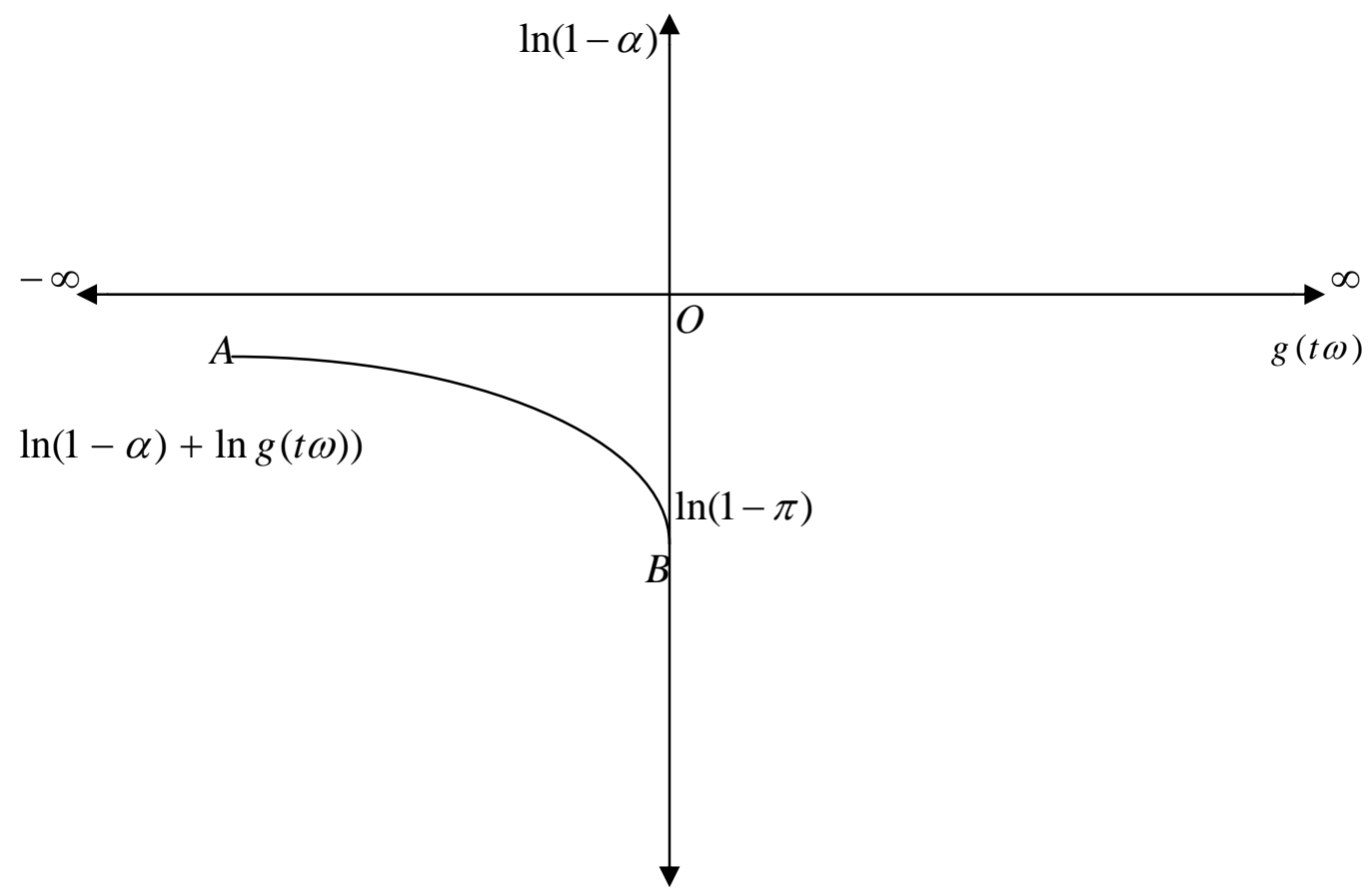


Taking these facts into account, curve $\mathrm{AB}$ in Figure $\mathbf{A} 2$ describes the likely shape of the constraint on the average rate of infection.

Given the general shape of the constraint and that of the iso-average infection curves, it follows that reducing $\phi^{*}$ cuts average infection if and only if the slope of the constraint curve AB is less than one in absolute value. That is, reducing $\phi^{*}$ will reduce average infection if it reduces $\ln (1-\alpha)$ by less than it reduces $\ln (g(t \omega))$. Since the change in $\ln (g(t \omega))$, as $\phi^{*}$ falls from $\phi_{1}$ to $\phi_{0}$, is infinitely larger than the change in $\ln (1-\alpha)$, effective NEPs will sometimes reduce average infection. But it does not follow that effective NEPs always reduce average

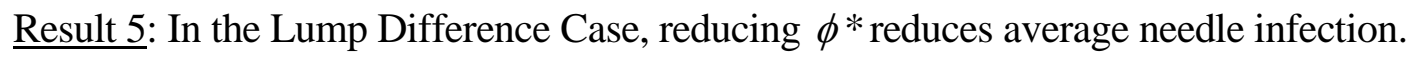

Proof: The lump difference case is given by

$$
\begin{array}{ll}
J(\phi)=L+(1-L) H(\phi) & \text { All } \phi \\
j(\phi)=(1-L) h(\phi) & \phi>\phi_{0}
\end{array}
$$

Noting that $\alpha=\frac{\pi\left(1-J\left(\phi^{*}\right)\right)}{q\left(\phi^{*}\right)}$, we get

$$
(1-\alpha)=\frac{1-\pi}{1-\pi L} \quad \text { for all } \phi^{*}
$$

Thus, the average constraint curve is flat (parallel to the $\ln (g(t \omega))$-axis) and the claim holds. Strict MLRP is the reason for the fall in $\alpha$ as $\phi^{*}$ rises, but only the weak MLRP holds in the interior of $\left[\phi_{0}, \phi_{1}\right]$ for the lump difference case: The IDUs who "switch" to new needles when $\phi^{*}$ falls are just the same as the IDUs who were already using new needles. 
Result 6: Let $x^{*} \cong 1.79$ be the solution to $e^{-x}\left(1+x+x^{2}\right)=1$. It can also be readily checked that for $x \in\left(0, x^{*}\right), e^{-x}\left(1+x+x^{2}\right)>1$ with the opposite holding true for $x>x^{*}$. If $t \omega>x^{*}$, then reducing $\phi^{*}$ always reduces average needle infection rates.

Proof: For reductions in $\phi^{*}$ to reduce average needle infection, we need

$$
\left|\frac{\partial}{\partial \phi^{*}} \ln g(t \omega)\right|>\frac{\partial}{\partial \phi^{*}} \ln (1-\alpha)
$$

From (A9) we have that

$$
\frac{\partial}{\partial \phi^{*}} \ln g(t \omega)=\frac{-g^{\prime}}{g} \frac{\partial(t \omega)}{\partial \phi^{*}}=\frac{-g^{\prime}}{g}\left[\frac{\pi j}{q}+\frac{\pi^{2} J j}{q^{2}}+\frac{\pi J(1-\pi) h}{q^{2}}\right]
$$

And from (A8), we have

$$
\frac{\partial}{\partial \phi^{*}} \ln (1-\alpha)=\frac{\pi j}{q}-\frac{\pi h(1-J)}{q(1-H)}
$$

Combining these with (A14) we have the following necessary and sufficient condition for reductions in $\phi^{*}$ to reduce average needle infection:

$$
h\left(\frac{1-J}{1-H}-\frac{1-\pi}{q} J \frac{g^{\prime}}{g}\right)>\frac{j}{g}\left(g+g^{\prime}\left(1+\frac{\pi J}{q}\right)\right)
$$

The derivation of (A15) used the result $\frac{\partial}{\partial \phi^{*}} \ln g(t \omega)>0$. Since $\frac{g^{\prime}}{g}<0$, the left-hand side of (A15) is always positive. Now consider the expression in brackets on the right-hand side and note that $\frac{\pi J}{q}$ is the argument of both $g($.$) and g^{\prime}($.$) .$ 
Thus we can set $x=\frac{\pi J}{q}$ and rewrite this expression as $g(x)+g^{\prime}(x)(1+x)$, which, in turn, equals $\frac{1}{x^{2}}\left[e^{-x}\left(1+x+x^{2}\right)-1\right]$. For $\frac{\pi J}{q}>x^{*}=1.79$, the right-hand side of (A15) is negative, so that the condition for reductions in $\phi^{*}$ to reduce average needle infection rates is met. QED

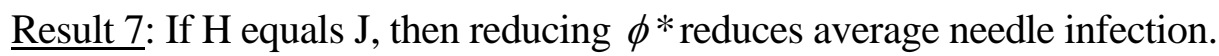

Proof: Let $\mathrm{H}=\mathrm{J}$ and, therefore, $\mathrm{h}=\mathrm{j}$ in (A15). Then the relevant necessary and sufficient condition for the result to hold becomes

$$
h\left(1-\frac{1-\pi}{q} J \frac{g^{\prime}}{g}\right)>j\left(1+\frac{g^{\prime}}{g}\left(1+\frac{\pi J}{q}\right)\right)
$$

Since $g^{\prime}($.$) is negative, the expression in brackets on the left-hand side of (A16) exceeds one.$ However, the expression in brackets on the right-hand side of (A16) is less than one, using similar reasoning. Thus (A16) holds. QED

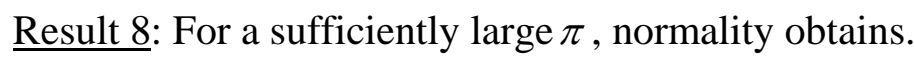

Proof: It is easy to see that for every $\phi^{*} \in \operatorname{int}\left[\phi_{0}, \phi_{1}\right], Z\left(\phi^{*}, \pi\right)$ is a continuous function of $\pi$ and $\phi^{*}$, with continuous derivatives and with $Z\left(\phi^{*}, 1\right)=H\left(\phi^{*}\right)$ \{Note that $\alpha=1$, whenever $\pi=1$, the average infection rate equals 1 , and the rest follows from the equation $Z\left(\phi^{*}, \pi\right)=H\left(\phi^{*}\right) \lambda$ \} When $\pi=1$ a decrease in $\phi^{*}$ leads to a decrease in $H\left(\phi^{*}\right)$ and, therefore $Z\left(\phi^{*}, 1\right)$. By continuity, there exists some $\bar{\delta}$ such that for all $\delta<\bar{\delta}$, reductions in $\phi^{*}$ also decrease $Z\left(\phi^{*}, 1-\bar{\delta}\right) \cdot \mathrm{QED}$ 


\section{TECHNICAL APPENDIX B}

Consider the following example. Let $\left[\phi_{0}, \phi_{1}\right]=[0,2]$ and

$$
\begin{aligned}
J(\phi)= & \operatorname{Min}(\phi, 1) & & \\
H(\phi) & =0.01 \phi, & & \text { for } \phi \leq 1 \\
& =0.01+0.99(\phi-1), & & \text { for } 1<\phi \leq 2
\end{aligned}
$$

It can be readily checked that this pair of functions satisfies both stochastic dominance and (weak) MLRP. Let $\pi=0.01$.

Suppose now that $\phi^{*}$ falls from 0.9 to 0.8 . Then it can be checked that the average needle infection rate $\lambda$ rises from 0.00514 to 0.00615 , so that needle response is not regular. The exposure $H\left(\phi^{*}\right)(1-\pi)$ of HIV-negative IDUs falls from 0.09 to 0.08 , but this decline is not sufficient to outweigh the increase in average needle infections. Thus, the rate of new human infections rises from 0.0000463 to 0.0000492 .

Notice that since terminal needle infection always falls when $\phi^{*}$ falls, changes in the terminal needle infection can tell us nothing about the direction of the change in $Z($.$) . In this$ example, terminal needle infection falls from 0.01013 (when $\phi^{*}=0.9$ ) to 0.01011 (the case where $\phi^{*}=0.8$ ). 


\section{TECHNICAL APPENDIX C}

(C1). Estimating the "average cost" of being infected with HIV for an injecting drug user (IDU)

We do this by (a) assessing the potential reduction in years lived by an HIV-negative IDU who gets infected with HIV; and (b) estimating the monetary value of those lost years.

C1.1. We will begin by estimating the potential reduction in years lived by an IDU owing to HIV infection. We construct life tables for IDUs with and without HIV, to estimate the expected reduction in life years lived owing to an HIV infection.

\section{Life Tables for IDUs without HIV}

Life tables for IDU (without HIV) are not exactly the same as life tables for the general population, because of the higher risk of mortality of the former. "Excess" mortality among IDUs arises because of a variety of conditions including, but not limited to, drug overdose, toxicity of drugs, cardiovascular problems (bacterial endocarditis), Hepatitis C, HPV, and a range of sexually transmitted conditions (CDUHR 2005).

The best information for constructing life tables for IDUs is likely to come from cohort studies, where the cohort is not contaminated with mortality from HIV-infections. However, sometimes experts working in the area have made their own informed decisions about mortality rates. We rely on both types of evidence.

- A 24-year cohort study of 581 narcotics addicts in California over the period 1962-1986 suggests an annual mortality rate of 1.25 percent among the addicted population with the average addict aged about 25 years at the time of entry into the study in 1962 (Hser et al. 2001).

- De Angelis et al. (2004; see also Kaplan 1989, Pollack 2001; Law et al. 2001) use an annual mortality rate among injecting drug users of 0.75 percent per year in their study that estimated incidence and prevalence of drug users in the United Kingdom. They also used age-varying annual mortality rates among drug users, starting from 0.32 percent for the age-group 15-24 years, to 1.07 percent for the age-group 45-54 years, for the period 1984-93, obtained from Ghodse et al. (1998). 
- Another estimate of mortality rates among IDU without HIV is available in Termorshuizen et al. (2005), with a cohort in Amsterdam. They estimate an annual average mortality rate of about 0.80 percent-1.01 percent over a 20-year period, beginning with a person's initiation into injecting drug use.

- A study by Galai et al. (2003) of the ALIVE cohort of injecting drug users in Baltimore estimated an overall mortality of 27.2 percent over the 12-year period from 1988-2000. This indicates a mortality rate on an annual basis of the order of 2.6 percent per year (0.026). However, a significant portion (35 percent) of this cohort was HIV-positive.

Taking these studies into account, we used two estimates of annual mortality rates for HIVnegative IDUs: a lower bound of 0.75 percent, and an upper bound of 1.25 percent. For arriving at a life table for an HIV-negative IDU, we assumed that until the age of 20 years (the age of initiation into injecting drug use) an IDU has the same survival probabilities as that of a member of the general population; he/she then experiences higher than average mortality rates ( $0.75 \%$ or $1.25 \%$ annually) for the rest of his life until (at a high enough age) the mortality in the general population reaches this level, whereupon an IDU begins to experience the same mortality as the general population - typically at an age of 75 years or older.

\section{Life Tables for IDU with HIV}

We need to calculate life tables separately (with and without HAART) given that IDUs, in at least some countries, may be able to access cheap drugs; and survival data in many cohort studies is complicated by the availability of HAART, since one might reasonably expect that IDUs with HIV live longer with HAART than without.

- Van Asten et al. (2003) suggest that HAART reduces mortality among HIV+ injecting drug users by about 28-36 percent, a number smaller than in the earlier literature of about 50-64 percent. If we assume, as per van Asten et al. (2003), that the median time period from infection until death is 10-12 years (or about 11 years) for an IDU with HIV, then we have that, in the absence of HAART, an HIV-positive IDU of say, 20 years of age 
would live for an additional median time of 11 years (an annual average mortality rate of roughly 6.1 percent -0.061$)$.

- Brettle et al. (1996) estimated a median survival time of 12.6 years of injecting drug users, over the period 1983-1995 in a cohort of injecting drug users in the United Kingdom. This would suggest an annual average mortality rate of about 5.3 percent (0.053) among HIV+ injecting drug users without HAART.

- A recent study among IDUs in British Columbia (Canada) by Lloyd-Smith et al. (2006, Table 3) estimated that expected additional years of survival at age 20 years was in the range of 23.0-24.5 (average 23.8) years for individuals on anti-retroviral therapy (HAART), or a mortality rate of about 0.041-0.043 annually.

Table C1 describes the expected years of life lived for an IDU (at age 20) with and without HIV, using life tables for HIV-positive IDUs constructed from these studies.

Table C1: Expected Years of Life for an IDU, with and without HIV

\begin{tabular}{|c|c|c|}
\hline \multirow{2}{*}{ Range of Estimates } & $\begin{array}{c}\text { HIV-Negative } \\
\text { Life Expectancy at 20 } \\
\text { (in years) }\end{array}$ & $\begin{array}{c}\text { HIV-positive } \\
\text { Life Expectancy at 20 } \\
\text { (in years) }\end{array}$ \\
\hline Low Mortality & 52.8 & 23.8 (HAART) \\
\hline High Mortality & 45.9 & 17.3 (No HAART) \\
\hline
\end{tabular}

Note: Although the number of mean years of survival for a person with HIV (but no HAART) appears somewhat on the "high" side, it should be noted that this corresponds to a median survival time of roughly 12 years.

This set of estimates suggests that an IDU with HIV aged 20 years can expect to live between 22.1 years and 35.5 years less than an IDU without HIV. 


\section{C.1.2. Expected Loss of Income from HIV Infection}

Next we constructed estimates of the "value" of life years lost by an IDU, owing to HIV infection. Two methods were used: (a) the human capital method; and (b) value of a statistical life.

C.1.2.a. Consider first the human capital method. In addition to the expected number of years lived (with and without HIV), we need four additional pieces of information (1) length of working life; (2) earnings when employed; (3) period of unemployment or incarceration; and (4) a rate of discount.

As for the first, we assumed that the IDU continues to work until he/she reaches the age of 65 years, or earlier, in case of death. According to the National Survey on Drug Use and Health (NSDUH) of 2005, the age at initiation in cocaine/heroin use is typically 20-22 years. It is possible that individuals move into injection drug use a few years after initiation. We do not make this distinction, and take that initiation into injection drugs occurs simultaneously along with entry into drug use.

Assuming an individual IDU to be currently 20 years of age (and, therefore, just initiated into injection drug use), we need to deduct from 45 (65 years less 20 years) the expected length of period for which he is likely to be incarcerated (the probability of being arrested times the length of arrest), or otherwise unemployed. There are several sources of data relating to employment and unemployment, some of which are probably not reflective of the conditions faced by injecting drug users.

- Consider the likelihood of being employed (or unemployed). Several studies assess the impact of substance use on the likelihood of being unemployed, hours of work, and productivity. However, the findings are mixed, and no clear conclusion emerges about the relationship between drug use and unemployment or earnings (e.g., French et al. 1998; Terza and Vechnak 2001; Zarkin et al. 1998). Indeed, there are also several studies that show that drug and alcohol use is either unrelated to earnings and work performance, or even positively, related. Common to almost all of these studies is that their definition of substance use/drug consumer often reflects a very low threshold for a drug user (e.g., reported consuming marijuana or another substance at least once in the last six months), 
in addition to methodological issues related to a possibly two-way causality between income/unemployment and drug use.

- A more recent study by DeSimone (2002b) used NLSY data for 1988 to assess the impact of marijuana and cocaine use on the likelihood of being employed. Nearly 93.7 percent of the sample population reported working at least one hour (the measure of employment!) in the previous year. Nonetheless, this study found cocaine use reduces the likelihood of being employed by 23-32 percent.

One might suspect that injecting drug users lie at one extreme of the drug consuming population and that estimated rates of unemployment in the econometric literature are downwardly biased owing to the low thresholds adopted for defining a drug user. For this reason, we focused more directly on socioeconomic information collected from cohort studies of injecting drug users, despite their small size and potential selection biases arising from subject recruiting methods. Analysis of data from these studies yield quite different answers regarding unemployment rates and earnings.

- In a study of more than 9,000 IDUs in nine cities in the United States, Williams et al. (1997) found unemployment rates of 65 percent.

- Another study, of 600 IDUs in Florida, found that nearly 55 percent were unemployed at the time of the interview (Chitwood et al. 1997).

- Estrada (1998) found, in another study of 760 IDUs in 16 United States cities, unemployment rates among non-white IDUs on the order of 80 percent and higher.

- Mandell et al. (1999) found, in a population of 500 African-American IDUs in Baltimore, unemployment rates that were even higher.

- Doherty (2000) found only 22 percent of a sample of 250 young IDUs to be employed in Baltimore.

Unfortunately, these studies did not always clarify whether the reported unemployment rates were contaminated by the presence of individuals who may also have been incarcerated during the reference period. They do, however, convey a picture of serious unemployment in the 
IDU population, much higher than that reported by econometric analyses on the subject. For the purposes of this paper, we work with an unemployment rate of 70 percent, reflecting both the likelihood of incarceration and being unable to find a job even when actively looking for it.

Now consider earnings when employed. Roughly 59 percent of the population in the United States belongs to the 20-65 year age group. A per capita GDP (in 2005) of US\$42,000 translates into a per-adult income in the 20-65 year age group of US\$70,000. Thus, with an unemployment rate of 70 percent (among IDU), this means an expected income of no more than US $\$ 21,000$ per IDU in any given year. Note that we are assuming that each IDU in the agegroup 20-65 years seeks to work and earns an income exactly equal to the average.

Now, an assumption of income of US\$21,000 per adult IDU may seem a bit high in the context of data from IDU cohort studies. For instance, Galai et al. (2003) in their analysis of African-American IDUs in Baltimore note that 75 percent of their sample had an annual (legal) income of less than US\$5,000. If we assume that the distribution of income among IDUs in Baltimore is exponentially distributed, we can easily check that the mean income of Baltimore IDUs is of the order of US\$3,606, substantially less than our estimate, based on GDP per adult. Another sample of IDUs (Estrada 1998) found that about 55\% of the respondents had an income of less than US\$6,000 annually. Again, assuming an exponential distribution of income, we get a mean income of US\$7,514. Finally, Chitwood et al. (2001) in their analysis of 600 IDUs in Florida found that 44 percent of their sample had an annual income of less than US\$10,000 (which using the assumption of exponential distribution of income, yields a mean IDU income of US\$12,523). Given that these published estimates are nearly 7-10 years old, adjusting these numbers for growth in nominal GDP per capita yield roughly an annual income range of US\$6,000 to US\$20,040 per IDU. Finally, noting that IDU also have “illegal” incomes from theft, sex work and drug sales, we end up with the conclusion that the figure of US\$21,000 as annual income per IDU may not be as unrealistic as may seem at first sight.

Using life-tables for IDU who are HIV-negative and life tables for IDU who are not, we estimated the lost income due to HIV-infection under the human capital method. We assume that per-adult real income grows at 2 percent per year, and that the discount rate is 52.5 percent (see below).

The expected lifetime earnings of an IDU who is HIV negative are roughly US\$76,000, according to our estimates. With HIV, the expected lifetime earnings would 
have been US\$69,400. With these adjustments, we assess that the expected lifetime loss of earnings from HIV infection is roughly US\$6,600 in present discounted terms for HIVnegative injecting drug users. This calculation does not include the cost of HAART, which most of the IDU are unlikely to be able to afford, nor for that matter, the cost of treating opportunistic infections. More than likely, such individuals will depend on emergency and social services for their medical and other care. Expenditures on these services will obviously matter for social cost-benefit analysis, although not from the perspective of the individual drug injector.

C.1.2.b. Estimating the value of statistical life years lost owing to HIV is generally considered a more attractive method to assess the cost of HIV infection to an IDU. We know very little about the value of a statistical life (or HIV infection) of an injecting drug user: that is, a measure of the willingness to pay for risk reduction in the current period (and hence increased length of life free of HIV infection in future periods), divided by a measure of reduction in risk of infection with HIV.

One way to do this is look at the price differential between a new needle in the black market and an old needle and then to inquire by how much individuals' risk of HIV infection is reduced by using a new needle, compared to an old needle. This would need the price of new needles, the price of old needles and the average rate of infection in an old needle. In general, the last is not an easy number to find. Alternately, one could use existing estimates of the value of a statistical life in the literature.

- Kniesner et al. (2005) used a large panel of American workers to conclude that the value of a statistical life is roughly on the order of US\$ 6 million (the mid-point of an estimated range of US\$5.3 million and US\$6.7 million). This cannot immediately be associated with the statistical value of life lost due to HIV infection by an IDU for two reasons: (a) IDUs have lower earnings than the average worker, and the value of a statistical life varies with income; and (b) deaths from HIV occur in the future, so discounting is necessary, and drug users may have higher discount rates than their non-drug using counterparts. 
Previously we saw that IDU annual incomes were of the order of US\$6,000 to US\$20,400 per person (an average of US\$13,000). Alternately, we could have chosen US\$21,000 as their annual income. The Kniesner et al. (2005) estimate of the value of a statistical life and early estimates reported in Viscusi and Aldy (2003) correspond to worker incomes in the range of US\$30,000-\$40,000. The question arises: What is the effect of this difference in earnings on the way an IDU might assess his/her statistical life?

As it turns out, Viscusi and Aldy summarize results of a very large number of crosscountry studies, in addition to carrying out estimates of their own, suggesting that the income elasticity of the value of a statistical life ranges between 0.5 and 0.6 (a mid-point of 0.55 ). Taking the incomes of IDUs as being half that of the workers reported in these studies, we have that the value of a statistical life (VSL) of an IDU is currently on the order of US\$4.35 million.

Based on this estimated value of a statistical life, we calculated the annualized value of a statistical life year for an IDU, using a standard annuitization formula (Viscusi and Aldy 2004). If VSLY is the value of a statistical life year (constant for the rest of his life) for a person of a specific age, $\mathrm{V}$ the value of a statistical life, " $\mathrm{r}$ " the rate of discount, and $\mathrm{L}$ the number of years this person can expect to live, we have that

$$
V S L Y=\frac{\left(\frac{r}{1+r}\right) V}{\left(1-(1+r)^{L}\right)}
$$

Available literature suggests that drug users have substantially higher discount rates than non-drug users (Bretteville-Jensen 1999; Kirby and Petry 2004). Indeed, an analysis of injecting drug users (current and former) in Oslo, Norway by Bretteville-Jensen (1999) suggests average annual discount rates of 90 percent among current users, 15 percent among past users, and only 5-percent among individuals who do not use drugs. For our purposes, we will work with a discount rate of 52.5 percent (the average of $90 \%$ and $15 \%$ ), given that our interest is in the injecting drug using population (although other discount rates can be considered). With this discount rate, we end up with a value of a statistical life year of US\$2.06 million, when mortality among IDUs (HIV-negative) was 0.75 percent and US\$2.12 million when mortality was $1.25 \%$. Lower discount rates would, of course, yield lower values of VSLY. 
We now define the statistical value of exactly one extra year lived, two extra years lived, three extra years lived, and so on. These simply correspond to the present discounted value of the number of years lived. From the life tables for IDUs (HIV-negative and HIV-positive (with and without HAART)), we can assess the present discounted value of life for each individual. This yields the loss to the IDU from being HIV-infected to range from US\$350,000 to US\$570,000 (or the average of $\$ 460,000$ ).

\section{C.2. Estimation of Exit Rates from HIV+ and HIV-IDU populations ( $\mu$ and $\eta$ ):}

There are at least three ways to exit: cessation of drug use, death, and incarceration. Moreover, death rates will differ for IDUs who are HIV-positive and those are not HIV-positive.

\section{C.2.1. Cessation Rate of IDUs}

Let us begin with "cessation" of drug use, which turns out not to be a straightforward notion. First, drug users can stop consuming drugs altogether in a one-shot transition from drug use to abstinence. In other cases, they may temporarily cease consuming drugs, only to relapse later. It is quite common also to find drug users transitioning in and out of drugs several times during their careers. The task is further made difficult by the varying definitions used by various authors to define "cessation" - e.g., no substance abuse in the last 4 months, no substance abuse in the last 6 months, and so on.

We estimate the cessation rate from existing cohort studies in the manner described below. First, for any given IDU cohort we inquire about the proportion of the cohort injecting at the end of the study/or at time of death/loss due to follow up, compared to baseline levels. The truncation of the data in case of loss due to follow up/death could downwardly bias the data towards a lower cessation rate if the people dying would have ceased to inject drugs later, or if people who could not be followed up consisted disproportionately of people who would have ceased to inject later. On the other hand, individuals injecting episodically may have had this pattern in the past, so that recording them as "full-time" IDU at the beginning of the study might upwardly bias the cessation rate.

- Galai et al. (2003) study of the 12-year ALIVE cohort suggests that the proportion of those currently using drugs fell from 1 to 0.54 at the end of the study period/or absence 
due to mortality/loss to follow up. Under the assumptions of the previous paragraph, this yields a cessation rate of 0.051 (or 5.1 percent) annually.

- A study for the United Kingdom (Robertson et al. 1994), suggested that over a 10-year period, 47 percent of an IDU cohort stopped injecting. That would suggest a cessation rate of about 0.063 (or 6.3 percent) annually.

- A 20-year follow-up of IDU in the Amsterdam cohort study (Termorshuizen et al. 2005) found, after adjusting for higher rates of abstinence in study dropouts, a cessation rate of 3.8 percent annually.

- Data from another study, of 85 narcotics addicts, not necessarily injectors, found a cessation rate of 1.6 percent over the period from 1985 to 1996. Other studies in the literature have used cessation rates ranging from 5 percent to 12 percent annually, although they have tended to focus on drug users, not necessarily IDUs (De Angelis et al. 2004).

For our analysis we used a cessation rate of IDU of 5 percent (0.05) annually, both for HIV+ as well as for HIV- individuals.

\section{C.2.2. Mortality among IDU}

Now consider death rates of injecting drug users, the second way in which IDUs can exit their population. These rates obviously differ for people who are HIV+, or not, and may also differ among HIV+ people for those who can access HAART and those who cannot. This has already been discussed in the context of life tables for IDUs. We will consider two scenarios of annual average mortality rates among HIV-negative IDUs: 0.75 percent, and 1.25 percent. The rationale for using these rates has been described earlier. We will assume that these rates apply to all HIV-negative IDUs in the population of interest, whether or not they cease injecting. For HIV-positive IDUs, an annual exit rate of 5\% was assumed.

\section{C.2.3. Incarceration Rates}

There is, first, the question of whether IDUs really stop injecting drugs in prison. If they do not, and there is considerable evidence for this, then there is no real need to worry about exits from 
the IDU population owing to incarceration. However, if incarceration does lead to net exits, be they HIV-positive, or HIV-negative IDUs, then it is important that we incorporate it in the model.

Suppose that individuals (IDUs) who enter prisons depart permanently from the druginjecting population. Then, to assess the exit rate from arrests, we want to ask the following questions to be consistent with the other data, relating to mortality and cessation of drug use: what is the likelihood of an individual IDU being jailed in any given year, and if jailed, what is the typical length of such incarceration? We need information on both because in cessation and mortality, the departure is on an annual basis. Throughout, we shall assume that HIV-negative and HIV-positive people have the same likelihood of being incarcerated as well as having the same duration of incarceration.

In general incarceration rates tend to be high among IDUs. In the ALIVE cohort of IDUs studied by Galai et al. (2003, p.698), the likelihood of incarceration in the 10 years prior to study entry was 67 percent, with similar findings being reported for another set of Baltimore IDUs by Doherty et al. (2000). Similarly, 78 percent of a cohort of IDUs in the United Kingdom reported being in prison at least once over a 10-year period (Robertson et al 1994), with little difference between HIV-positive and HIV-negative IDUs in the proportion incarcerated.

One way to proceed would be to assume that the probability of being arrested at least once in any given year is statistically independent of the probability of being arrested in any other year. Then given (from above) that the cumulative probability of being arrested at least once over a period of 10-years is 67-78 percent, we end with up with the likelihood of an IDU being arrested, followed by conviction and imprisonment, in any given year, of 0.11 to 0.14 (or 11-14 percent). The most recent data from the Bureau of Justice Statistics (http://www.ojp.usdoj.gov/bjs/sent.htm) suggests that the average length of incarceration in state prisons for drug possession (the most likely offense) is about 14 months. From this we take the average length of imprisonment to be one year - and end up with an exit rate of IDUs of 0.110.14 each year on account of incarceration (likelihood of being imprisoned times the length of imprisonment).

In general, the assumption that the likelihood of being arrested is statistically independent across different years is difficult to justify. Moreover, it is possible that some IDUs are being released from incarceration, even as others are being newly imprisoned, so that that the net exit rate from the HIV-positive (or negative) IDU population as a consequence is zero in steady-state IDU 
imprisonment. Thus, a 33-year longitudinal study of IDUs found that about 19.2 percent of the (currently alive) cohort was in prison at the time of the interview in 1985 and 14.0 percent (of the then-alive cohort) was in prison at the time of interview in 1996 - percentages that are not too dissimilar.

For our analysis we took a value of zero net exits due to incarceration, although other possibilities exist.

\section{C.2.3.1. Entry Rates of HIV-negative individuals into IDU population $(\varepsilon)$}

From the United States National Household Survey on Drug Use for 2004 and 2005, we obtained point estimates of persons "ever having injected drugs". These came to 3.506 million in 2004 and 3.678 million in 2005. Taking these point estimates as essentially the "truth", this yields a net incidence of IDU (in the sense of having injected at least once in the last year) of 0.172 million for the year 2005. One possibility is to think of this as the entry rate into IDU populations. However, this calculation ignores the possibility that some lifetime users of injection drugs may have died in the preceding year, or otherwise exited. If incarceration is in steady state, we can conveniently ignore any departures/entrants due to incarceration (or release). That leaves us with (a) mortality and cessation from hard-core IDU activity as the major sources of exit (note: there is the possibility that not all of the 0.172 million are really hard-core IDUs of the kind we are concerned with in this paper).

We adopted an alternative approach to figure out exit rates from the HIV- and HIV+ IDU populations. The UN estimate of IDUs in the United States was 1.3 million for 2004 (Aceijas et al. 2004). If this was a steady-state outcome, applying an annual mortality rate of 1.0 percent to 80 percent of this population (assuming 20 percent were HIV-positive - the mid-point of the range of estimates in Aceijas et al. (2004)) yields an estimate of 10,400 HIV-negative drug injectors who died last year. An additional 5\% depart due to cessation of drug use, amounting to a total of 62,400 exits in the HIV-negative IDU population.

Assuming a 5\% annual mortality rate in the HIV-positive IDU population, and an additional 5\% of exits due to cessation of drug use, yields 26,000 exits from the HIV-positive IDU population (excluding any exits due to conversion of status from HIV-negative to HIVpositive). This yields a total of 88,400 total exits annually. In steady state, this also means entry of 88,400 (0.088 million) new HIV-negative IDUs. This is not too far from the estimates of the 
National Household Survey on Drug Use estimates of new injectors in the last year (of 0.172 million). 


\section{TECHNICAL APPENDIX D}

The Kaplan Model (also Kaplan and O’Keefe 1993)

Suppose that there are $\mathrm{N}$ shared needles and $\mathrm{P}$ drug injectors. Let $\mathrm{N} / \mathrm{P}=\gamma$. At time $t, n(t)$ is the proportion of needles that are infected, and $p(t)$ is the proportion of the drug-injecting population that is infected. Infected needles and the infected people are just random elements of their respective pools. At every moment, a constant fraction $\varepsilon$ of IDUs is selected to (randomly) share needles. Sorting is purely random: infected needles are neither more nor less likely to get matched with infected people than non-infected needles are; and the converse is true as well.

Assume $\frac{\gamma}{\varepsilon}(=\mathrm{N} / \varepsilon \mathrm{P})$ is less than one so every needle always finds a (single) user. Then the probability that a person is matched with an infected needle is $\frac{m(t)}{\varepsilon}$; the probability that a needle is matched with an infected person is $p(t)$. An uninfected person using an infected needle becomes infected with probability $\tau$. Infected people depart from the drug-injecting population at the rate $\mu$ per unit time, and are replaced on a one-for-one basis by uninfected new arrivals. Similarly, an NEP catches randomly chosen needles (like a mosquito net) and replaces them with uninfected new needles. The rate at which the NEP catches needles is given by $\kappa$. In the absence of an NEP, $\kappa$ equals zero and needles live forever. Let $\theta$ be the rate at which needles are cleaned with bleach, or otherwise become less infectious owing to the limited lifespan of the virus in the needle, prior to injecting. In view of this, the equations of motion are:

$$
\begin{aligned}
& \dot{p}(t)=\frac{[1-p(t)](1-\theta) \gamma n(t) \tau}{\varepsilon}-\mu p(t) \\
& \dot{n}(t)=[1-n(t)] p(t)-\kappa n(t)-[\theta(1-p(t))] n(t)
\end{aligned}
$$


Kaplan and O'Keefe (1993) use the above model to evaluate the impact of the New Haven needle exchange on HIV transmission among injecting drug users. They estimate the parameters $\gamma$, $\varepsilon, \tau, \theta$ and $\mu$, which they then assume remain constant, and simulate the equation system under two alternative assumptions about values for $\kappa$ : first that $\kappa$ equals zero, and second that $\kappa$ is equal to the value observed in the New Haven NEP. They compare the number of new infections of humans that occur in the two simulations, and conclude that the New Haven NEP reduced new infections by about a third.

For our purposes, it suffices to look at steady states. It is easy to show that the equation system (D1-D2) has two steady states, one of which is $p(t)=n(t)=0$, but only one of them is stable. Let $\alpha=\frac{\gamma \tau}{\varepsilon}$ and suppose

(D3) $\alpha(1-\theta)-(\kappa+\theta) \mu>0$

Then the steady state $(0,0)$ is unstable and the unique (globally) stable steady state is

(D4) $\bar{n}=\frac{\alpha(1-\theta)-(\kappa+\theta) \mu}{\alpha(1+\kappa)(1-\theta)}$

(D5) $\bar{p}=\frac{\alpha(1-\theta) \bar{n}}{\alpha(1-\theta) \bar{n}+\mu}$

(D6) $\bar{p}=\frac{\alpha(1-\theta)-\mu \theta-\mu \kappa}{(\alpha+\mu)(1-\theta)}$ 